\title{
The clusters Abell 222 and Abell 223: a multi-wavelength view ${ }^{\star}$
}

\author{
F. Durret ${ }^{1,2}$, T. F. Laganá ${ }^{3}$, C. Adami ${ }^{4}$, and E. Bertin ${ }^{1,2}$ \\ 1 UPMC Université Paris 06, UMR 7095, Institut d'Astrophysique de Paris, 75014 Paris, France \\ e-mail: durret@iap.fr \\ 2 CNRS, UMR 7095, Institut d'Astrophysique de Paris, 75014 Paris, France \\ 3 IAG, USP, R. do Matão 1226, 05508-090. São Paulo/SP, Brazil \\ ${ }^{4}$ LAM, Pôle de l'Étoile Site de Château-Gombert, 38 rue Frédéric Joliot-Curie, 13388 Marseille Cedex 13, France
}

Accepted 30 March 2010 / Received 7 May 2010

\begin{abstract}
Context. The Abell 222 and 223 clusters are located at an average redshift $z \sim 0.21$ and are separated by 0.26 deg. Signatures of mergers have been previously found in these clusters, both in X-rays and at optical wavelengths, thus motivating our study. In X-rays, they are relatively bright, and Abell 223 shows a double structure. A filament has also been detected between the clusters both at optical and X-ray wavelengths.

Aims. We analyse the optical properties of these two clusters based on deep imaging in two bands, derive their galaxy luminosity functions (GLFs) and correlate these properties with X-ray characteristics derived from XMM-Newton data.

Methods. The optical part of our study is based on archive images obtained with the CFHT Megaprime/Megacam camera, covering a total region of about $1 \mathrm{deg}^{2}$, or $12.3 \times 12.3 \mathrm{Mpc}^{2}$ at a redshift of 0.21 . The X-ray analysis is based on archive XMM-Newton images. Results. The GLFs of Abell 222 in the $g^{\prime}$ and $r^{\prime}$ bands are well fit by a Schechter function; the GLF is steeper in $r^{\prime}$ than in $g^{\prime}$. For Abell 223, the GLFs in both bands require a second component at bright magnitudes, added to a Schechter function; they are similar in both bands. The Serna \& Gerbal method allows to separate well the two clusters. No obvious filamentary structures are detected at very large scales around the clusters, but a third cluster at the same redshift, Abell 209, is located at a projected distance of $19.2 \mathrm{Mpc}$. X-ray temperature and metallicity maps reveal that the temperature and metallicity of the X-ray gas are quite homogeneous in Abell 222, while they are very perturbed in Abell 223.

Conclusions. The Abell 222/Abell 223 system is complex. The two clusters that form this structure present very different dynamical states. Abell 222 is a smaller, less massive and almost isothermal cluster. On the other hand, Abell 223 is more massive and has most probably been crossed by a subcluster on its way to the northeast. As a consequence, the temperature distribution is very inhomogeneous. Signs of recent interactions are also detected in the optical data where this cluster shows a "perturbed" GLF. In summary, the multiwavelength analyses of Abell 222 and Abell 223 are used to investigate the connection between the ICM and the cluster galaxy properties in an interacting system.
\end{abstract}

Key words. galaxies: clusters: individual: Abell 222 - X-rays: galaxies: clusters - galaxies: clusters: individual: Abell 223 galaxies: clusters: intracluster medium

\section{Introduction}

Galaxy evolution is known to be influenced by environmental effects, which are particularly strong in galaxy clusters, all the more when they are undergoing merging events. The analysis of galaxy luminosity functions (GLF), and in particular of their faint-end slopes is a good way to trace the history of the faint galaxy population and the influence of merging events on this population. As summarized for example in Table A.1 of

* Based on observations obtained with MegaPrime/MegaCam, a joint project of CFHT and CEA/DAPNIA, at the Canada-France-Hawaii Telescope (CFHT) which is operated by the National Research Council (NRC) of Canada, the Institut National des Sciences de l'Univers of the Centre National de la Recherche Scientifique (CNRS) of France, and the University of Hawaii. This work has also made use of data products produced at TERAPIX and the Canadian Astronomy Data Centre as part of the Canada-France-Hawaii Telescope Legacy Survey, a collaborative project of NRC and CNRS. The X-ray analysis is based on XMM-Newton archive data. This research has made use of the NASA/IPAC Extragalactic Database (NED) which is operated by the Jet Propulsion Laboratory, California Institute of Technology, under contract with the National Aeronautics and Space Administration.
Boué et al. (2008), this slope can strongly vary from one cluster to another, and from one region to another in a given cluster.

Superseding the dynamical analyses of Proust et al. (2000) and Girardi \& Mezzetti (2001), Dietrich et al. (2002) have obtained a redshift catalogue for 183 galaxies in the region of the cluster pair Abell 222/223, out of which 153 belong to one of the two clusters. Their analysis was also based on photometry in the $V$ and $R$ bands. They have estimated redshifts of $0.2126 \pm 0.0008$ and $0.2079 \pm 0.0008$, and velocity dispersions of 1014 and $1032 \mathrm{~km} \mathrm{~s}^{-1}$, for Abell 222 and Abell 223 respectively. As indicated by Dietrich et al. (2002) these velocity dispersions are somewhat higher than those expected from the X-ray luminosities. Abell 222 appears relatively relaxed, with a galaxy velocity histogram consistent with a Gaussian. On the other hand, the adaptive kernel galaxy density map of Abell 223 shows two peaks, though surprisingly the Dressler-Schectman test (Dressler \& Schectman 1988) does not find these two peaks and the DIP statistics cannot reject the null hypothesis of a unimodal distribution, implying that a Gaussian parent population cannot be excluded. Dietrich et al. (2002) derived respective mass to light ratios of $202 \pm 43$ and $149 \pm 33 h_{70} M_{\odot} / L_{\odot}$ and luminosity functions in the $R$ band down to absolute magnitudes of -19.5 , and 
Table 1. Summary of the observations.

\begin{tabular}{lrr}
\hline \hline Filter & $g^{\prime}$ & $r^{\prime}$ \\
\hline Number of coadded images & 5 & 8 \\
Exposure time $(\mathrm{s})$ & 2000 & 4000 \\
Seeing (arcsec) & 0.81 & 0.73 \\
Limiting magnitude $(5 \sigma)$ & 26.7 & 26.4 \\
\hline
\end{tabular}

found that a bridge of galaxies seems to exist between the two clusters. Note that such bridges of galaxies joining two clusters are still quite rare, another example at a comparable redshift being the filament detected between Abell 1763 and Abell 1770 by Fadda et al. (2008).

A weak lensing analysis of the Abell 222/223 system was performed by Dietrich et al. (2005), who found evidence for a possible dark matter filament connecting both clusters. They interpret the difference in redshift between Abell 222 and Abell 223 as implying a separation along the line of sight of $15 \pm 3 h_{70}^{-1} \mathrm{Mpc}$. Their weak lensing surface mass density contours again show evidence for a double structure in Abell 223.

The existence of a 1.2 Mpc wide filament between both clusters was also found in X-rays by Werner et al. (2008).

We present here a muti-wavelength optical and X-ray analysis of the clusters Abell 222 and Abell 223. The optical part is based on imaging in the $g^{\prime}$ and $r^{\prime}$ bands obtained with the CFHT Megaprime/Megacam camera and retrieved from the Megapipe image stacking pipeline at CADC (Gwyn 2009). As seen in Table 1 , the $r^{\prime}$ band data are about 0.55 mag deeper than the $g^{\prime}$ band data (by taking an average color $g^{\prime}-r^{\prime}=0.85$ ), and will be given priority in the morphological analysis; the $g^{\prime}$ data will be used to select bright cluster members from color-magnitude diagrams, and also because the $g^{\prime}$ band is more sensitive to star formation than the $r^{\prime}$ band. These images are deeper than those of Dietrich by at least 0.5 mag (our raw galaxy counts start decreasing for $r^{\prime}>24.5$ while Dietrich et al. say that their counts in the $R$ band stop following a power law for $R>24$ ), and therefore allow to probe the GLF to somewhat fainter magnitudes. Our X-ray analysis is based on archive XMM-Newton data.

We will consider hereafter that the two clusters have a common redshift of 0.21 , for which Wright's cosmology calculator (Wright 2006) gives a distance of $1035 \mathrm{Mpc}$, a spatial scale of $3.427 \mathrm{kpc} / \mathrm{arcsec}$ and a distance modulus of 40.07 (assuming a flat $\Lambda \mathrm{CDM}$ cosmology with $H_{0}=70 \mathrm{~km} \mathrm{~s}^{-1} \mathrm{Mpc}^{-1}, \Omega_{\mathrm{M}}=0.3$ and $\left.\Omega_{\Lambda}=0.7\right)$.

The paper is organised as follows. We describe our optical analysis in Sect. 2, and results concerning the galaxy luminosity function in Sect. 3. A search for substructures in both clusters based on the Serna-Gerbal method is described in Sect. 4. The X-ray data analysis and results, including temperature and metallicity maps, are presented in Sect. 5. An overall picture of this cluster pair is drawn in Sect. 6.

\section{Optical data and analysis}

\subsection{The optical data}

We have retrieved from the CADC Megapipe archive (Gwyn 2009) the reduced and stacked images in the $g^{\prime}$ and $r^{\prime}$ bands (namely G002.024.493-12.783.G and G002.024.49312.783.R) and give a few details on the observations in Table 1. Observations were made at the CFHT with the Megaprime/Megacam camera, which has a pixel size of $0.186 \times$ $0.186 \operatorname{arcsec}^{2}$.

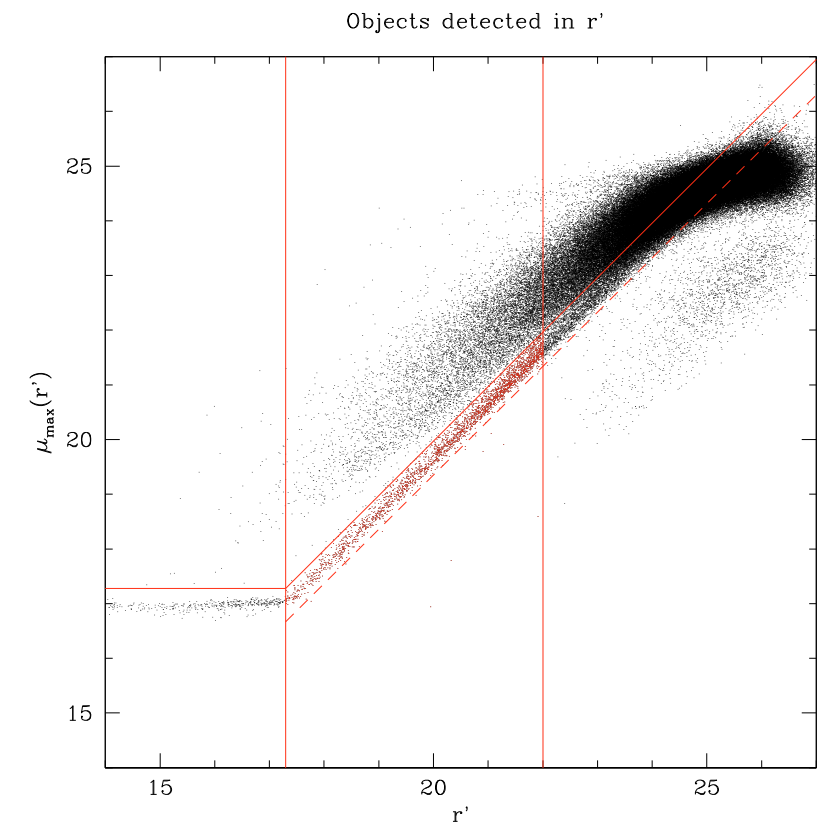

Fig. 1. Central surface brightness in the $r^{\prime}$ band as a function of $r^{\prime}$ magnitude. The horizontal and oblique full red lines separate the galaxies (above these lines) from the stars (below these lines). The oblique dotted red line shows the limit for stars computed for $17.25<r^{\prime}<22$ (see text). The two vertical lines correspond to $r^{\prime}=17.25$ where the stars stop being saturated, and $r^{\prime}=22$, where the fit to calculate the star sequence was limited (see text).

We did not use the catalogues available for these images, because they were made without masking the surroundings of bright stars, so we preferred to build masks first, then to extract sources with SExtractor (Bertin \& Arnouts 1996). Due to the dithering pattern, the total area covered by the $r^{\prime}$ image was slightly larger than the Megacam $1 \mathrm{deg}^{2}$ field: $1.196 \mathrm{deg}^{2}$.

Objects were detected and measured in the full $r^{\prime}$ image, then measured in the $g^{\prime}$ image in double image mode. Magnitudes are in the $\mathrm{AB}$ system. The objects located in the masked regions were then taken out of the catalogue, leading to a final catalogue of 223414 objects. All these objects have measured $r^{\prime}$ magnitudes, and 214669 also have measured $g^{\prime}$ magnitudes.

Since the $r^{\prime}$ catalogue is deeper, and the seeing is also better in this band (see Table 1), we will perform our star-galaxy separation in the $r^{\prime}$ band.

\subsection{Star-galaxy separation}

In order to separate stars from galaxies, we plotted the maximum surface brightness $\mu_{\max }$ in the $r^{\prime}$ band as a function of $r^{\prime}$. The result is shown in Fig. 1.

The best fit to the star sequence visible in Fig. 1 calculated for $17.25<r^{\prime}<22$ is $\mu_{\max }=0.996 * r^{\prime}-0.247$, with standard deviations on the slope and constant of 0.002 and 0.047 respectively. The point-source (hereafter called "star") sequence is clearly visible for $r^{\prime}<22$, with the star saturation showing well for $r^{\prime}<17.25$. We will define galaxies as the objects with $\mu_{\max }\left(r^{\prime}\right)>17.25$ for $r^{\prime}<17.25$, and as the objects above the line of equation $\mu_{\max }=0.996 * r^{\prime}-0.247+0.3$, or $\mu_{\max }=0.996 * r^{\prime}+0.053$. Stars will be defined as all the other objects (see Fig. 1). The small cloud of points observed in Fig. 1 under the star sequence is in fact defects, but represents less than $2 \%$ of the number of stars. We thus obtain a star and a galaxy catalogue. 
As a check to see up to what magnitude we could trust our star-galaxy separation, we retrieved the star catalogue from the Besançon model for our Galaxy (Robin et al. 2003) in a $1 \mathrm{deg}^{2}$ region centered on the position of the image analysed here. Such a catalogue is in AB magnitudes (as ours) and is corrected for extinction. In order for it to be directly comparable to our star catalogue, we corrected our star catalogue (and our galaxy catalogue as well, for later purposes) for extinction: 0.071 mag in $g^{\prime}$ and $0.052 \mathrm{mag}$ in $r^{\prime}$ (as derived from the Schlegel et al. 1998 maps).

The $r^{\prime}$ magnitude histogram of the objects classified as stars in our $r^{\prime}$ image roughly agrees with the Besançon star catalogue for $r^{\prime} \leq 20$, though in some bins our counts tend to be somewhat higher than those of the Besançon model even for bright magnitudes. For $r^{\prime}>20$, we detect more stars than predicted by the Besançon model (the difference is about 35\% at $r^{\prime} \sim 21$ ). This could imply that our star-galaxy separation is not valid for $r^{\prime}>20$; however, as suggested by Fig. 1, this is a rather bright limit, as confirmed by simulations adapted to match comparable data, where we found that the star-galaxy separation determined with this method was reliable at least up to $r^{\prime}=21$ (Boué et al. 2008). In reality, the disagreement between our star counts and those predicted by the Besançon model is most probably due to the fact that our field is located in the direction of one of the densest regions of the Sagittarius stream (Ibata et al. 2001; Yanny et al. 2009). It is therefore normal to observe more stars than predicted by the Besançon model, which does not take this stream into account. Note that there may also be a small contribution due to quasars and AGN, since their number at $r^{\prime} \sim 20.5$ is expected to be about 20 per square degree (see Richards et al. 2006, Fig. 13).

For $r^{\prime}>20$, we will conservatively consider that the stargalaxy separation may be wrong, and we will compute galaxy counts by counting the total number of objects (galaxies plus stars) per bin of $0.5 \mathrm{mag}$, and considering that the number of galaxies is equal to the total number of objects minus the number of stars predicted in each bin by the Besançon model. As mentioned below, we have checked that the galaxy number counts in the $20<r^{\prime}<22$ range estimated by both methods (i.e. our stargalaxy separation and the subtraction of the Besançon counts to the total number of objects) are consistent within error bars.

Since the clusters are quite distant, they do not cover the whole field, so we extracted from the star and galaxy catalogues two catalogues as large as possible corresponding to the two clusters. We defined the J2000.0 positions of Abell 222 and Abell 223 as coinciding with the brightest galaxy of each cluster, i.e. $24.3921,-12.9912$ and $24.5099,-12.7575$ respectively (in degrees). Note that these are not exactly the cluster positions given by the NED database. The maximum possible radius to obtain independent catalogues for the two clusters was $0.1290 \mathrm{deg}$, or $1.6 \mathrm{Mpc}$ at a redshift of 0.21 .

For the Abell 222 and Abell 223 clusters separately, we thus obtained three complementary catalogues (with $g^{\prime}$ and $r^{\prime}$ mag): objects classified as galaxies (classification valid at least for $r^{\prime} \leq 20$ ), objects classified as stars, and a complete catalogue of galaxies+stars which will be used for $r^{\prime}>20$. The positions of the galaxies in the regions of the two clusters are shown in Fig. 2.

\subsection{Catalogue completeness}

The completeness of the catalogue is estimated by simulations. For this, we add "artificial stars" (i.e. 2-dimensional Gaussian profiles with the same Full-Width at Half Maximum as the average image Point Spread Function) of different magnitudes to

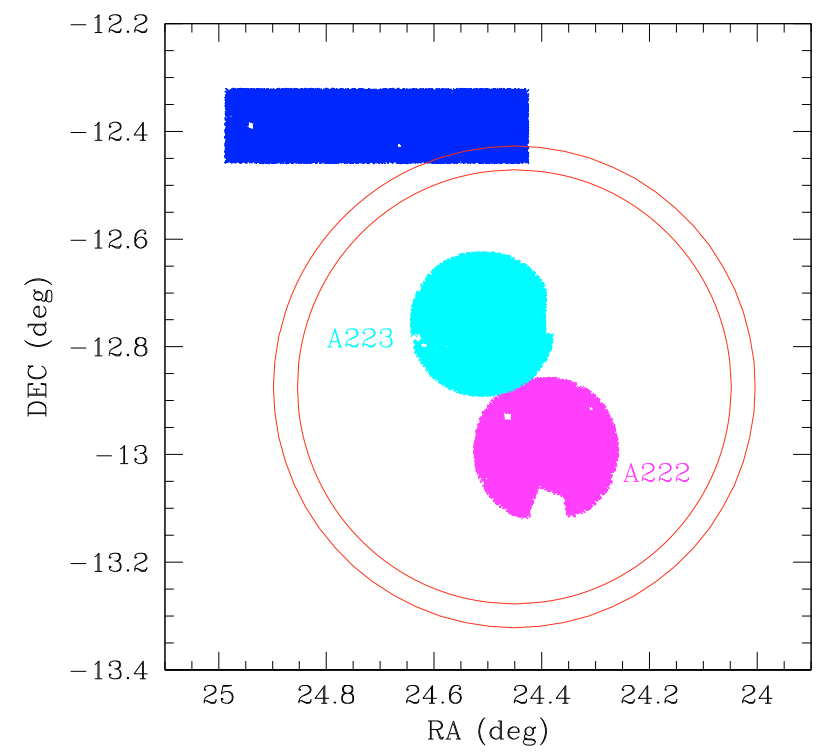

Fig. 2. Positions of the objects (stars and galaxies) in the Abell 222 (magenta) and Abell 223 (cyan) catalogues. The objects used to estimate background counts (see text) are shown in blue for the north rectangle and in red for the annulus surrounding the clusters. Note that the figure covers $1.2 \times 1.2 \mathrm{deg}^{2}$, and is slightly larger than the images, which cover $1.04 \times 1.15 \mathrm{deg}^{2}$.

the CCD images and then attempt to recover them by running SExtractor again with the same parameters used for object detection and classification on the original images. In this way, the completeness is measured on the original images.

In practice, we extract from the full field of view two subimages, each $1500 \times 1500$ pixels $^{2}$, corresponding to the positions of the Abell 222 and Abell 223 clusters on the image.

In each subfield, and for each $0.5 \mathrm{mag}$ bin between $r^{\prime}=20$ and 27, we generate and add to the image one star that we then try to detect with SExtractor, assuming the same parameters as previously. This process is repeated 100 times for each of the two fields and bands.

Such simulations give a completeness percentage for stars. This is obviously an upper limit for the completeness level for galaxies, since stars are easier to detect than galaxies. However, we have shown in a previous paper that this method gives a good estimate of the completeness for normal galaxies if we apply a shift of $\sim 0.5$ mag (see Adami et al. 2006). Results are shown in Fig. 3.

From these simulations, and taking into account the fact that results are worse by $\sim 0.5$ mag for mean galaxy populations than for stars, we can consider that our galaxy catalogue is complete to better than $80 \%$ for $g^{\prime} \leq 25$ and $r^{\prime} \leq 24.5$.

\subsection{Galaxy counts}

The surfaces covered by the Abell 222 and 223 catalogues (after excluding masked regions) are $0.0429 \mathrm{deg}^{2}$ and $0.0475 \mathrm{deg}^{2}$ respectively. Galaxy counts were computed in bins of 0.5 mag normalized to a surface of $1 \mathrm{deg}^{2}$.

For $r^{\prime} \leq 20$, galaxy counts were derived directly by computing histograms of the numbers of galaxies in the Abell 222 and Abell 223 catalogues. For $r^{\prime}>20$, we built for each cluster histograms of the total numbers of objects (galaxies+stars) and obtained galaxy counts by subtracting the numbers of stars predicted by the Besançon model. The resulting galaxy counts will 

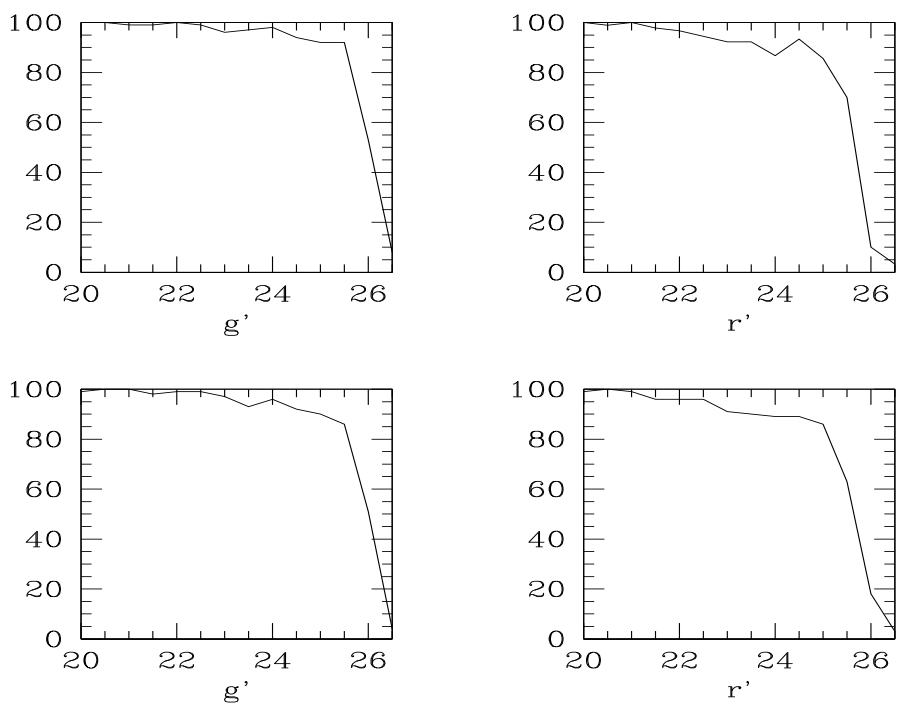

Fig. 3. Point source completeness as a function of magnitude in percentages for Abell 222 (top) and Abell 223 (bottom) in $g^{\prime}$ (left) and $r^{\prime}$ (right) for point-like objects (see text).

be used in the next section to derive the GLFs for both clusters in both bands.

As a test, we considered the galaxy counts in the four 0.5 mag bins for $20<r^{\prime}<22$ computed for both clusters with the two methods (i.e. first method: considering that the star-galaxy separation is valid, and second method: considering the total number of objects (galaxies+stars) and subtracting the number of stars predicted by the Besançon model to obtain the number of galaxies). In all cases, the differences are smaller than $15 \%$ (and in most cases they are only a few percent). Therefore, the limit (between $r^{\prime}=20$ and 22) at which we consider that our star-galaxy separation is not valid any more will not strongly influence our results.

Note that no k-correction was applied to the galaxy magnitudes.

\section{Results: colour-magnitude diagrams and galaxy luminosity functions}

In order to compute the galaxy luminosity functions of the two clusters, it is necessary to subtract to the total galaxy counts the number counts corresponding to the contamination by the foreground and background galaxies.

For galaxies brighter than $r^{\prime}=20$ we will select galaxies with a high probability to belong to the clusters by drawing colour-magnitude diagrams and selecting galaxies located close to this relation. A few spirals may be missed in this way, but their number in any case is expected to be small, as explained at the end of Sect. 3.1 (also see e.g. Adami et al. 1998). For galaxies fainter than $r^{\prime}=20$ we will subtract galaxy counts statistically.

\subsection{Colour-magnitude diagrams}

The mean values for $g^{\prime}-r^{\prime}$ are 0.85 and 0.87 for Abell 222 and Abell 223. The $g^{\prime}-r^{\prime}$ vs. $r^{\prime}$ colour-magnitude diagrams are shown in Fig. 4 for the two clusters. A sequence is well defined for galaxies in the magnitude range $18<r^{\prime}<22$ in both clusters. We computed the best fit to the $g^{\prime}-r^{\prime}$ vs. $r^{\prime}$ relations in this magnitude range by applying a linear regression (separately for the two clusters, since their colour-magnitude relations may not
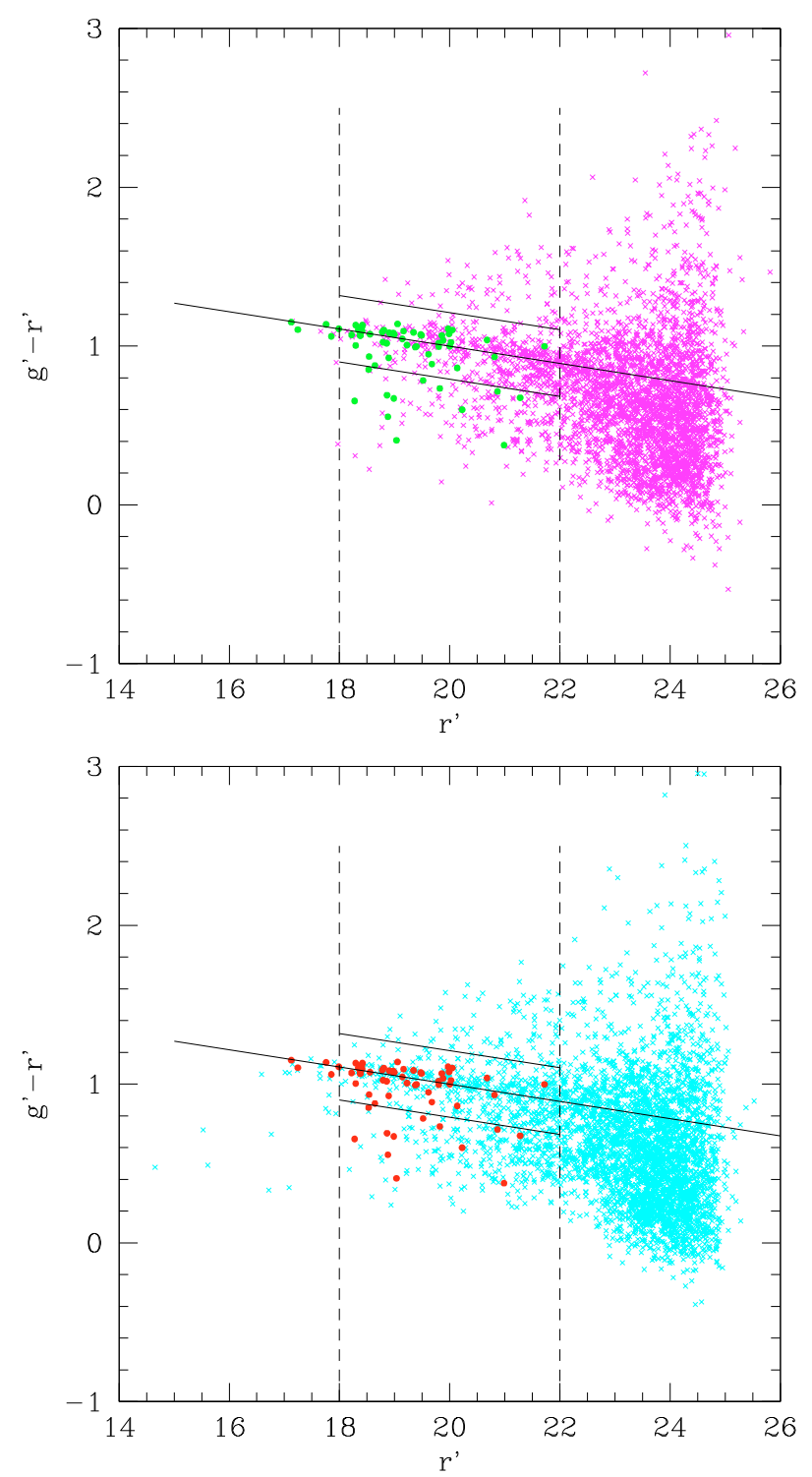

Fig. 4. $\left(g^{\prime}-r^{\prime}\right)$ vs. $r^{\prime}$ colour-magnitude diagrams for Abell 222 (top) and Abell 223 (bottom) for objects classified as galaxies from the $\mu_{\max }$-magnitude relation. The vertical dashed lines indicate the magnitude interval where the colour-magnitude relation was computed. The long oblique line shows the colour-magnitude relation, while the two short oblique lines indicate an interval of $\pm 3 \sigma$ around the colour-magnitude relation. The filled circles show the galaxies belonging to the clusters according to their spectroscopic redshifts.

be absolutely identical). We then eliminated the galaxies located more than $3 \sigma$ away from this relation and computed the $g^{\prime}-r^{\prime}$ vs. $r^{\prime}$ relations again.

The equations of the colour-magnitude relations are found to be: $g^{\prime}-r^{\prime}=-0.0545 r^{\prime}+2.091$ with $3 \sigma=0.21$ for Abell 222, and $g^{\prime}-r^{\prime}=-0.0474 r^{\prime}+1.945$ with $3 \sigma=0.21$ for Abell 223 .

For $r^{\prime}<20$, we will consider hereafter that all the galaxies located within $3 \sigma$ of these relations (i.e. between the two black lines of Fig. 4) belong to the clusters. With this condition, there are 141 and 144 galaxies belonging to Abell 222 and Abell 223 respectively with $r^{\prime} \leq 20$.

We also plot in Fig. 4 the galaxies belonging to the clusters according to their spectroscopic redshifts, i.e. galaxies in the $[0.195,0.215]$ redshift interval (see Sect. 4). We can see that their positions agree well with the colour-magnitude selection. 
For both clusters, we have estimated the number of blue cluster galaxies lost by selecting galaxies in the red sequence interval in the following way. First, we computed histograms of numbers of galaxies in the red sequence and below the red sequence in bins of 1 absolute magnitude in the $r^{\prime}$ band. The bins of interest here are $M_{r^{\prime}}=-21.5$ and -20.5 (see Fig. 4), roughly corresponding to $r^{\prime}=18.5$ and 19.5 . Then we estimated the number of foreground galaxies expected. Since the comoving volume at $z=0.21$ is $2.622 \mathrm{Gpc}^{3}$ (Wright 2006), and each of our clusters covers an area of $0.0522 \mathrm{deg}^{2}$ on the sky, the volume in the direction of each cluster is $3318 \mathrm{Mpc}^{3}$. By using the $R$ band luminosity function by Ilbert et al. (2005) in the [0.05-0.20] redshift bin (see their Fig. 6 and Table 1), we find that the percentages of "lost" galaxies are of the order of $10 \%-25 \%$ for $M_{r^{\prime}}=-20.5$ and of $10 \%$ for $M_{r^{\prime}}=-21.5$.

\subsection{Comparison field}

In order to perform a statistical subtraction of the background contribution for $r^{\prime}>20$, we considered several possibilities.

The most obvious solution would be to extract the background in an annulus surrounding the clusters. The largest outer circle that can be extracted, centered on the middle position between the two clusters $(24.4510,-12.8744 \mathrm{~J} 2000.0$ in degrees), has a radius of $0.4472 \mathrm{deg}$, or $5.55 \mathrm{Mpc}$. We can then take an inner circle of radius $0.4028 \mathrm{deg}$, or $5 \mathrm{Mpc}$. This annulus is drawn in Fig. 2. However, the number counts in this circle are higher than the CFHLTS Deep survey counts (see below), probably because the annulus is too close to the clusters and still includes cluster galaxies, so we will not use these counts as a background.

The Canada-France-Hawaii Telescope Legacy Survey (CFHTLS) has been taken with the same telescope, camera and filters as the data we are analyzing. The Deep survey explores a solid angle of $4 \times 1 \mathrm{deg}^{2}$ of the deep Universe, in four independent fields (http: //www. cfht . hawaii.edu/Science/ CFHLS/). Observations are carried out in five filters $\left(u *, g^{\prime}, r^{\prime}, i^{\prime}\right.$ and $z^{\prime}$ ) providing catalogs of sources that are $80 \%$ complete up to $i_{A B}=26.0$ (see http://terapix.iap.fr/cplt/oldSite/ Descart/CFHTLS-T0005-Release.pdf). We only consider the Deep survey here, because we are interested in the GLFs down to faint magnitudes. For the four deep fields, we computed the galaxy number counts in bins of $0.5 \mathrm{mag}$, in $g^{\prime}$ and $r^{\prime}$, normalized to a surface of $1 \mathrm{deg}^{2}$. The galaxy counts in these four fields are drawn in Fig. 5. As discussed before (see e.g. Boué et al. 2008, and references therein), the counts in these four fields somewhat differ, due to cosmic variance, and in particular the counts in the Deep 4 field are higher than in the other three. We will therefore take as background galaxy counts the average of the Deep 1, Deep 2 and Deep 3 field counts.

For comparison, we also extracted galaxy counts from a region assumed to be representative of the background in our image. This region was chosen to be a rectangle north of the clusters, in an area devoid of bright stars. It is shown as a blue rectangle in Fig. 2 and covers an effective area of $0.06825 \mathrm{deg}^{2}$. The corresponding counts (hereafter the "local" background counts) appear to be notably lower than the CFHTLS-Deep counts (Fig. 5); this may be the case if this region corresponds to a void located between the large scale filaments that converge towards the clusters.

Although we consider that the CFHTLS-Deep counts probably represent better the galaxy background counts, we will also fit the GLFs with these "local" background counts, keeping in mind the fact that these "local" counts are likely to overestimate
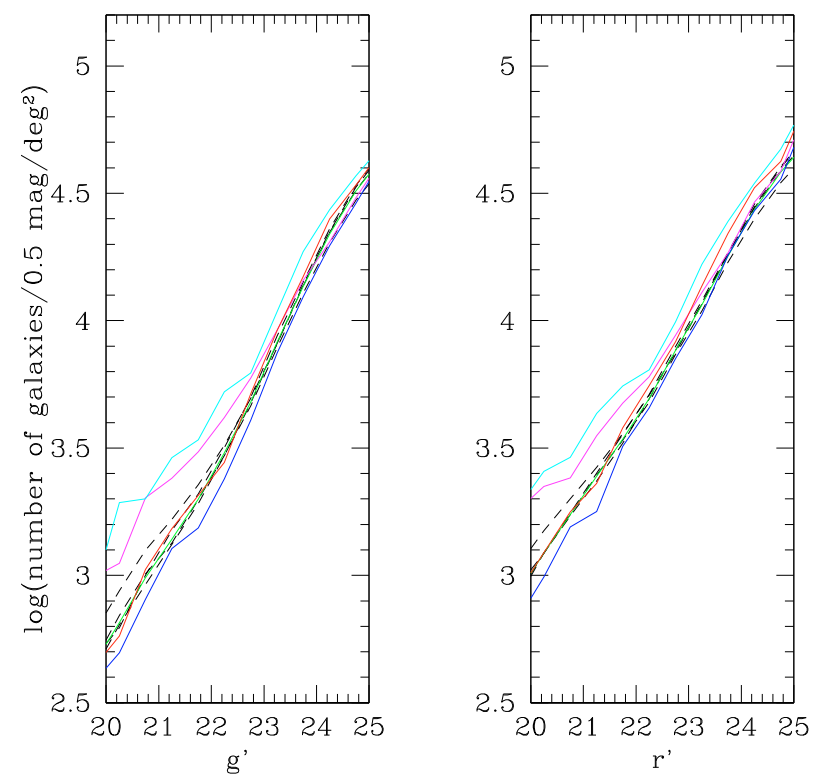

Fig. 5. Galaxy counts in the $g^{\prime}$ (left) and $r^{\prime}$ (right) bands for magnitudes $r^{\prime}>20$ where the background must be subtracted statistically, in logarithmic scale. The counts in Abell 222 and 223 are drawn in magenta and cyan respectively. The black dashed lines show the galaxy counts from the CFHTLS Deep survey, and the full green line shows the average of the Deep 1, Deep 2 and Deep 3 field counts. The blue and red lines correspond to the "local" backgrounds extracted from the same images as our clusters (red: in the annulus between the two circles of Fig. 2, blue: in the north rectangle, see text and Fig. 2). Error bars are Poissonian and are not plotted for clarity.

the GLFs thus derived for Abell 222 and 223. This will illustrate the difficulty to determine GLF parameters unambiguously.

We can note from Fig. 5 that galaxy counts are notably higher in Abell 223 than in Abell 222, implying that the former cluster is richer than the latter.

\subsection{Galaxy luminosity functions}

The Galaxy Luminosity Functions (GLFs) of Abell 222 and Abell 223 were calculated in bins of $0.5 \mathrm{mag}$ and normalized to $1 \mathrm{deg}^{2}$, as described above. We subtracted the background contribution using as background galaxy counts: 1) the average of the three Deep 1, Deep 2 and Deep 3 fields; 2) the "local" counts in the north blue rectangle of Fig. 2.

The GLFs are displayed for Abell 222 and Abell 223 in Figs. 6 and 7 respectively. The error bars drawn in these figures were taken to be 4 times the Poissonian errors on galaxy counts, as suggested by detailed simulations previously performed by our team for similar data (see Boué et al. 2008, Fig. 5).

The GLFs (as a function of absolute magnitude) were fit by a Schechter function:

$S(M)=0.4 \ln 10 \phi^{*} y^{\alpha+1} \mathrm{e}^{-y}$

with $y=10^{0.4\left(M^{*}-M\right)}$.

The parameters of the Schechter function fits of the GLFs are respectively given in Tables 2 and 3 for the two different background subtractions: the average of the CFHTLS Deep fields and the "local" background. The absolute magnitude range considered is indicated for each fit. The fits are drawn in Figs. 6 and 7 for Abell 222 and Abell 223 respectively.

If we compare the results given in Tables 2 and 3, we see that the $\Phi^{*}$ and $M^{*}$ parameters depend little on the background 
Table 2. Schechter parameters for galaxy luminosity functions (background galaxy counts taken from the CFHTLS Deep field counts).

\begin{tabular}{lccccc}
\hline \hline Cluster & Filter & Range & $\Phi^{*}$ & $M^{*}$ & $\alpha$ \\
\hline Abell 222 & $g^{\prime}$ & {$[-22.5,-16.0]$} & $2891 \pm 134$ & $-20.04 \pm 0.06$ & $-0.60 \pm 0.05$ \\
& $r^{\prime}$ & {$[-23.5,-16.0]$} & $937 \pm 64$ & $-22.01 \pm 0.07$ & $-1.43 \pm 0.01$ \\
Abell 223 & $g^{\prime}$ & {$[-25.5,-15.5]$} & $1017 \pm 72$ & $-21.05 \pm 0.06$ & $-1.30 \pm 0.02$ \\
& $r^{\prime}$ & {$[-26.0,-15.0]$} & $1107 \pm 62$ & $-22.05 \pm 0.06$ & $-1.29 \pm 0.01$ \\
\hline
\end{tabular}

Table 3. Schechter parameters for galaxy luminosity functions ("local” background galaxy counts).

\begin{tabular}{lccccc}
\hline \hline Cluster & Filter & Range & $\Phi^{*}$ & $M^{*}$ & $\alpha$ \\
\hline Abell 222 & $g^{\prime}$ & {$[-22.0,-16.0]$} & $2269 \pm 133$ & $-20.32 \pm 0.06$ & $-0.94 \pm 0.03$ \\
& $r^{\prime}$ & {$[-23.0,-16.0]$} & $1132 \pm 63$ & $-21.85 \pm 0.06$ & $-1.42 \pm 0.01$ \\
Abell 223 & $g^{\prime}$ & {$[-25.0,-15.0]$} & $819 \pm 55$ & $-21.20 \pm 0.05$ & $-1.42 \pm 0.01$ \\
& $r^{\prime}$ & {$[-26.0,-15.0]$} & $1042 \pm 58$ & $-22.10 \pm 0.06$ & $-1.34 \pm 0.01$ \\
\hline
\end{tabular}
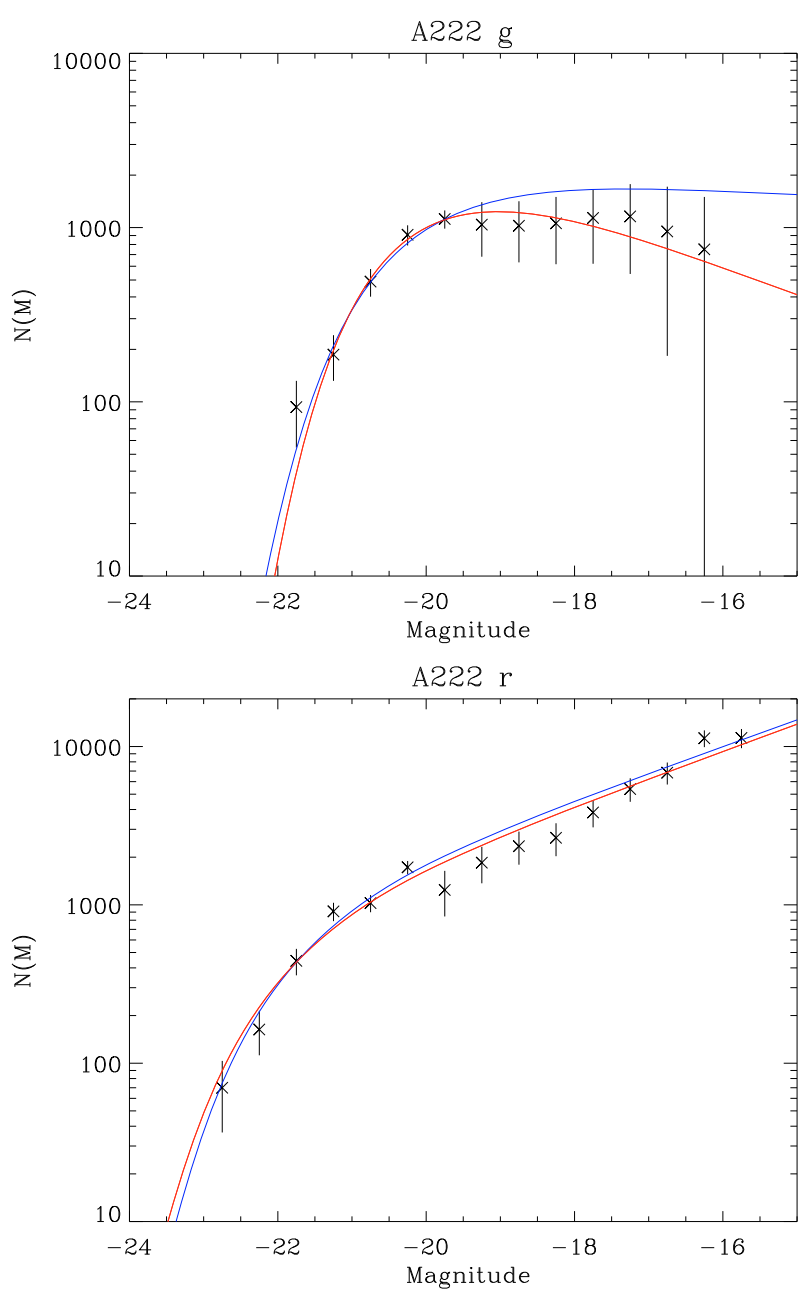

Fig. 6. Galaxy luminosity functions for Abell 222 in the $g^{\prime}$ (top) and $r^{\prime}$ (bottom) bands, in logarithmic scale. The pooints correspond to the subtraction of the background counts taken from the CFHTLS. Error bars are 4 times the Poissonian errors on galaxy counts (see text). The best Schechter function fits are drawn in red for the subtraction of background counts taken from the CFHTLS and in blue for "local" background counts (see text).

galaxy counts chosen. On the other hand, some variations are found in the faint end slope $\alpha$, as expected since it is at faint magnitudes that the background galaxy subtraction has a strong influence.
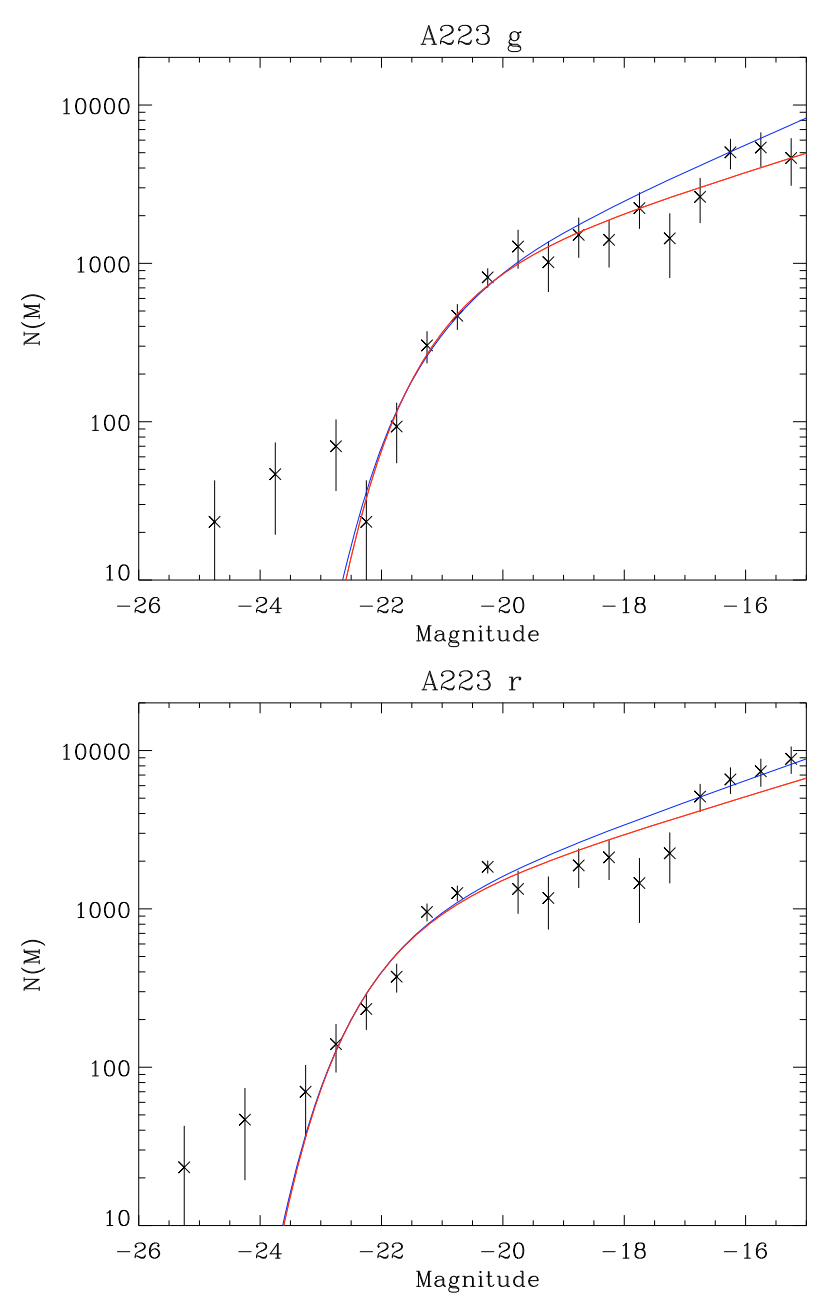

Fig. 7. Same as Fig. 6 for Abell 223.

For Abell 222, the GLF is quite well fit by a Schechter function in the $r^{\prime}$ band, with a faint end slope $\alpha=-1.43$ (the same for both background subtractions). On the other hand, the GLF is surprisingly flat in $g^{\prime}$ (slope $\alpha=-0.60$ or -0.94 , depending on background subtraction).

A Schechter function is obviously not sufficient to fit the GLFs of Abell 223, both in $g^{\prime}$ and $r^{\prime}$ : Abell 223 has more very bright galaxies than Abell 222, and a second component at bright magnitudes is obviously required. At faint magnitudes, the GLF slopes in $g^{\prime}$ and $r^{\prime}$ are comparable for Abell 223. They vary by 


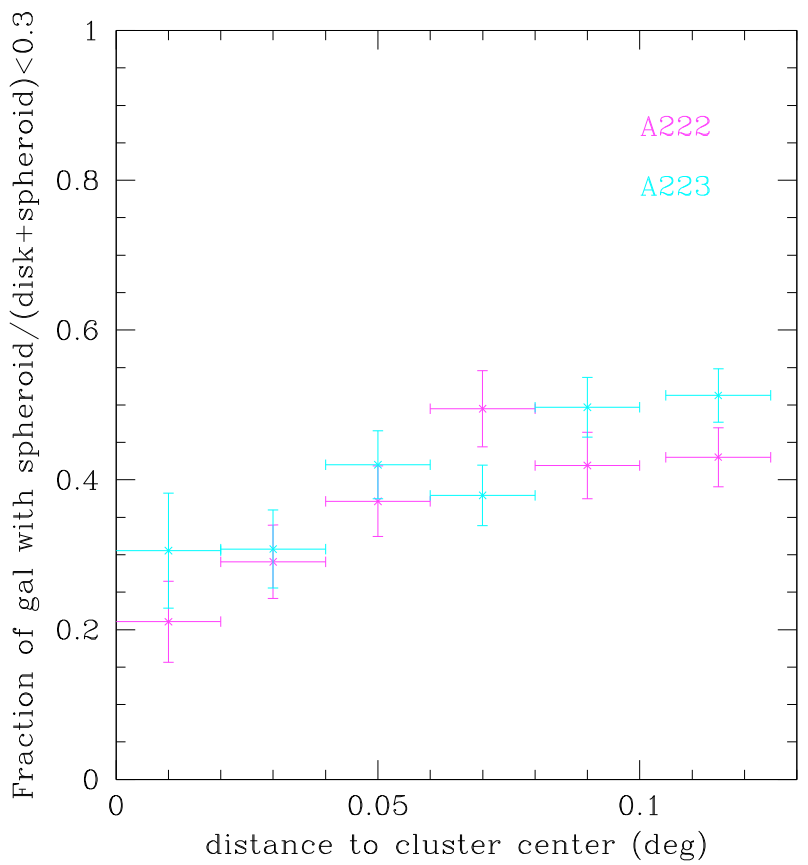

Fig. 8. Fraction of disk-dominated galaxies as a function of radius for Abell 222 (in magenta) and Abell 223 (in cyan).

small quantities ( 0.12 and 0.05 respectively) between one galaxy background subtraction and the other. However, these slopes are only indicative, since as seen in Fig. 7 the GLFs show strong wiggles for absolute magnitudes between -20 and -17 in $r^{\prime}$ and between -20 and -16 in $g^{\prime}$. Since Abell 223 is itself a double cluster (Dietrich et al. 2002), it is not surprising to find that it has a "perturbed" GLF. This is also the case for example for the Coma cluster, known to have a two-component GLF (Biviano et al. 1995).

If we compare the GLFs of the two clusters, we can see that they are more or less comparable in the $r^{\prime}$ band (except for the wiggles in Abell 223), while in the $g^{\prime}$ band the GLF is notably steeper in Abell 223 than in Abell 222. An explanation for this can be that both clusters have comparable old galaxy populations, but that the number of faint star forming galaxies is higher in Abell 223, where star formation can be triggered by recent interactions.

If we compare these GLFs to those found by other authors, we can note that the bright part of the GLF Schechter fits $\left(r^{\prime}<18\right.$, or $\left.M_{r^{\prime}}<-18.9\right)$ for both clusters are very similar in shape to the GLFs recently obtained e.g. by Andreon et al. (2008); these authors analyzed the GLFs of a sample of clusters at various redshifts, limited to relatively bright absolute magnitudes: $M_{V}<-19$.

The $\alpha$ slopes of the faint ends of the GLFs derived here (except for that of Abell 222 in the $g^{\prime}$ band) are within the broad range of values estimated by previous authors for different clusters, cluster regions and photometric bands (see e.g. the compilation in Table A.1 of Boué et al. 2008).

Note that Abell 222 and Abell 223 are at redshift 0.21, and few GLFs are available for clusters at such redshifts. Andreon et al. (2005) found somewhat shallower slopes for three clusters at redshifts $\sim 0.3$, but in the $K$ band, so the comparison with our results is not straightforward.

\subsection{Morphological segregation}

We applied a new tool developed in SExtractor that calculates for each galaxy the respective fluxes in the bulge (spheroid) and disk. This new experimental SEXTRACTOR feature fits to each galaxy a two-dimensional model comprised of a de Vaucouleurs spheroid (bulge) and an exponential disk. Briefly, the fitting process is very similar to that of the GalFit package (Peng et al. 2002) and is based on a modified Levenberg-Marquardt minimisation algorithm. The model is convolved with a supersampled model of the local Point Spread Function (PSF), and downsampled to the final image resolution. The PSF model used in the fits was derived with the PSFEx software (Bertin et al. 2010, in preparation) from a selection of $5436\left(g^{\prime}\right) / 6922\left(r^{\prime}\right)$ point source images. The PSF variations were fit using a 6th degree polynomial of $x$ and $y$ image coordinates.

The model fitting was carried out independently in the $g^{\prime}$ and $r^{\prime}$ bands. The bulge to total ratios discussed here were extracted from the red channel, limited to $r^{\prime}=22$, since beyond this magnitude results may become unreliable.

We applied this tool to look for evidence for morphological segregation, by computing the fraction of galaxies with a spheroid to total flux smaller than 0.3 , as a function of radius (i.e. in concentric circles of step $0.02 \mathrm{deg}$ ). The result is plotted in Fig. 8. In each bin, $1 \sigma$ errors were computed as follows: if $n$ is the number of galaxies having a spheroid to total flux smaller than 0.3 and $N$ the total number of galaxies, we assume that $n$ and $(N-n)$ are independent variables; in this case, the error on $n / N$ is:

$(n / N) \sqrt{(1 / n-1 / N)}$

We can see that there is a general increase of the fraction of diskdominated galaxies with radius as expected (see e.g. Biviano et al. 1997).

In order to see if there was a morphological segregation that could be linked to the presence of cosmological filaments, we computed that same fraction in angles of $45 \mathrm{deg}$ all around each cluster. The result is shown in Fig. 9, where angular sectors are numbered from 1 to 8 clockwise from east (see Fig. A.1). No strong trend is found in this plot within error bars, as expected if there is no obvious cosmological filament linking the clusters with the surrounding cosmic web. We can note however that for both clusters there is a dip in the fraction of bulgedominated galaxies in sector 6 , which corresponds to the line joining the two clusters; the filament already detected by Werner et al. (2008) using wavelet-decomposition in the [0.5-2] keV energy band is located along this same direction. In this zone the gas is not yet hot enough to influence the galaxies. If we look at Fig. 9 from Laganá et al. (2009), $2 \mathrm{keV}$ is the lower limit where ram-pressure plays an important role in galaxy clusters. Thus we would expect to detect a trend only if the filament was hotter than $2 \mathrm{keV}$.

Another possibility for this dip in the fraction of bulgedominated galaxies is a higher number of blue galaxies in which star formation was triggered due to ongoing collisions. Poggianti et al. (2004) argued that there is a striking correlation between the positions of the young and strong post-starburst galaxies and substructure in the hot intra-cluster medium (ICM) identified from XMM-Newton data, with these galaxies lying close to the edges of two infalling substructures. This result suggests that the interaction with the dense ICM could be responsible for the triggering of the star formation. 


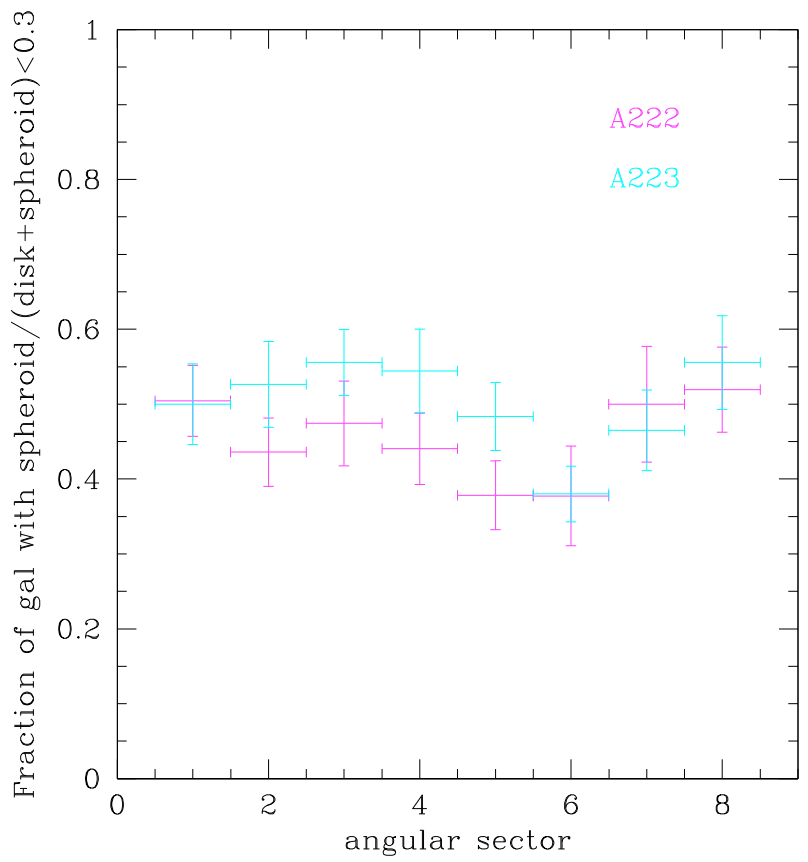

Fig. 9. Fraction of disk-dominated galaxies in sectors of 45 deg within each cluster, with the same colours as in the previous figure. Angular sectors are numbered from 1 to 8 clockwise from east (see Fig. A.1).

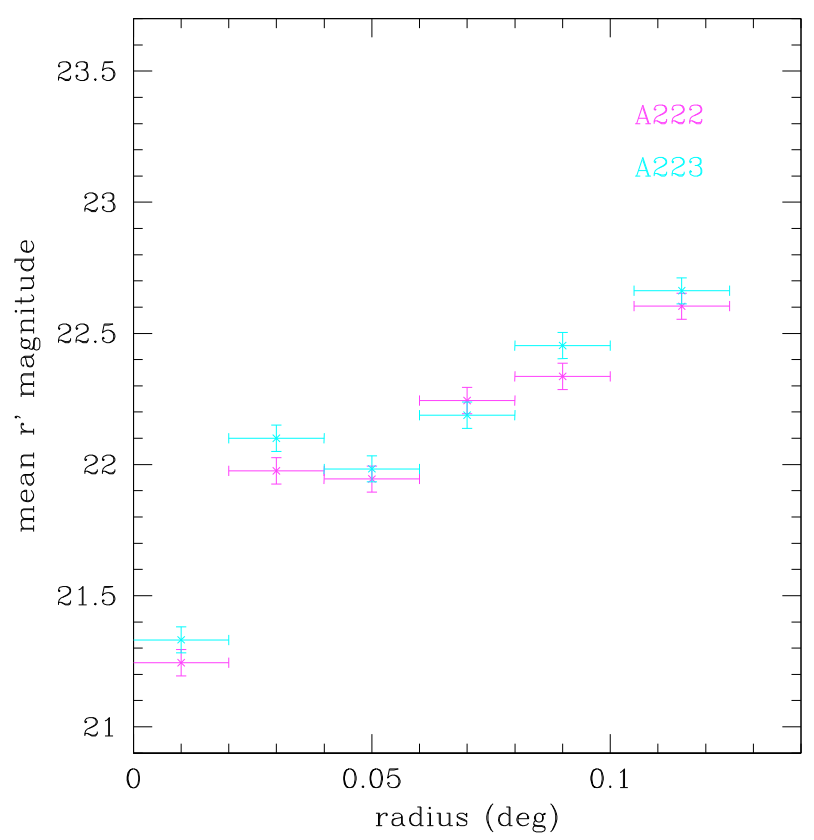

Fig. 10. Average $r^{\prime}$ magnitude as a function of radius for Abell 222 (in magenta) and Abell 223 (in cyan).

\subsection{Luminosity segregation}

We searched for luminosity segregation in both clusters by selecting galaxies brighter than $r^{\prime}=24$ and within the red sequence defined in Fig. 4, to increase the probability that they belong to the clusters. The mean $r^{\prime}$ magnitude was calculated in each of the radial bins previously defined and results are shown in Fig. 10. We observe an increase of the mean magnitude with radius: it is about 21.3 in the central bin, and then increases with radius from values around 22 to 22.6 .

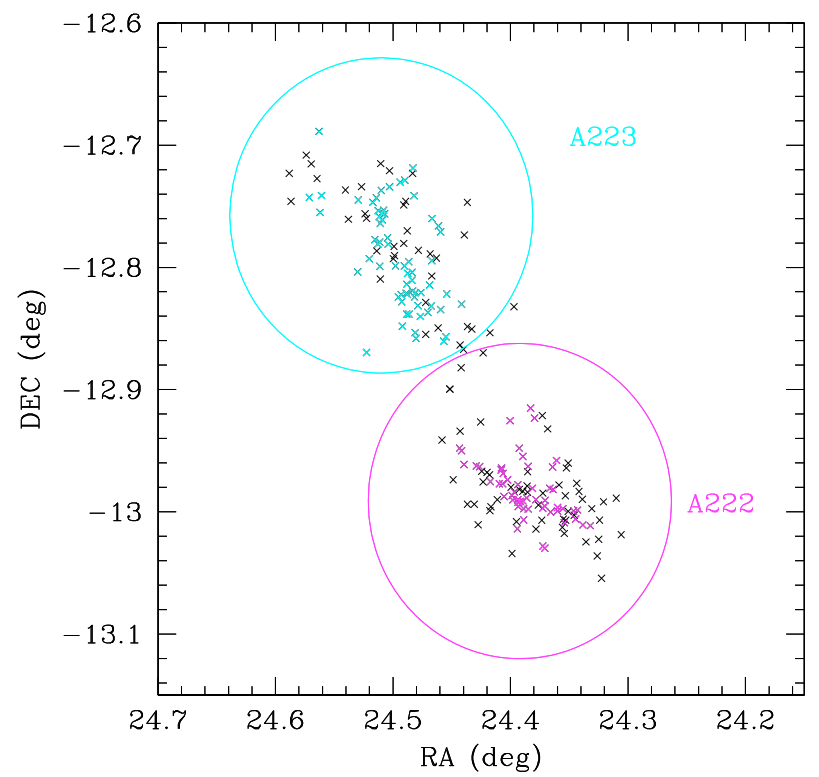

Fig. 11. Positions of the galaxies with measured redshifts and magnitudes in the regions covered by Abell 222 and Abell 223 (black crosses). The galaxies belonging to the two main gravitationally bound systems identified with the SG method are shown in colours (see text).

\section{Cluster-scale to large-scale structure}

We searched the NED database for galaxies with redshifts available in a very large region of 3 deg radius around Abell 222/223 and found 980 galaxies. This radius corresponds to $37 \mathrm{Mpc}$ at the distance of the studied clusters, i.e. to the typical diameter of a "bubble" or of a void in the universe (see e.g. Hoyle \& Vogeley 2004). This catalogue of galaxies with measured redshifts will be used first to search for substructures in the zones covered by our clusters, and second to search for structures (such as filaments) at a larger scale.

\subsection{Search for gravitationally bound structures}

Within the regions of our images covered by Abell 222 and Abell 223, 246 galaxies have measured redshifts, and 210 galaxies have both measured redshifts and magnitudes. Out of these 210 galaxies, 173 have redshifts in the $[0.18,0.24]$ interval. The positions of the 210 galaxies with measured redshifts and magnitudes are displayed in Fig. 11. It is interesting to note that, as traced by these galaxies, both clusters appear to be strongly elongated, with elongation position angles that differ from one cluster to the other. This strongly suggests that the clusters are not in dynamical equilibrium, in agreement with previous studies (see above, and also Zabludoff et al. 1995; Roettiger et al. 1996; Boschin et al. 2004; Girardi et al. 2006).

The corresponding redshift histogram, zoomed on the cluster redshift interval, is shown in Fig. 12.

We applied to the catalogue of 210 galaxies with redshifts and magnitudes the Serna \& Gerbal (1996) algorithm (hereafter $\mathrm{SG}$, which allows to search for substructures, separate galaxies forming gravitationally bound structures, and estimate the masses of these substructures. The dendogram thus obtained is shown in Fig. 13. It clearly shows two dynamically distinct substructures, corresponding to the two clusters: Abell 222 in the top half and Abell 223 in the bottom half. The two main gravitationally bound structures corresponding to these two clusters respectively include 55 and 64 group members. Their 


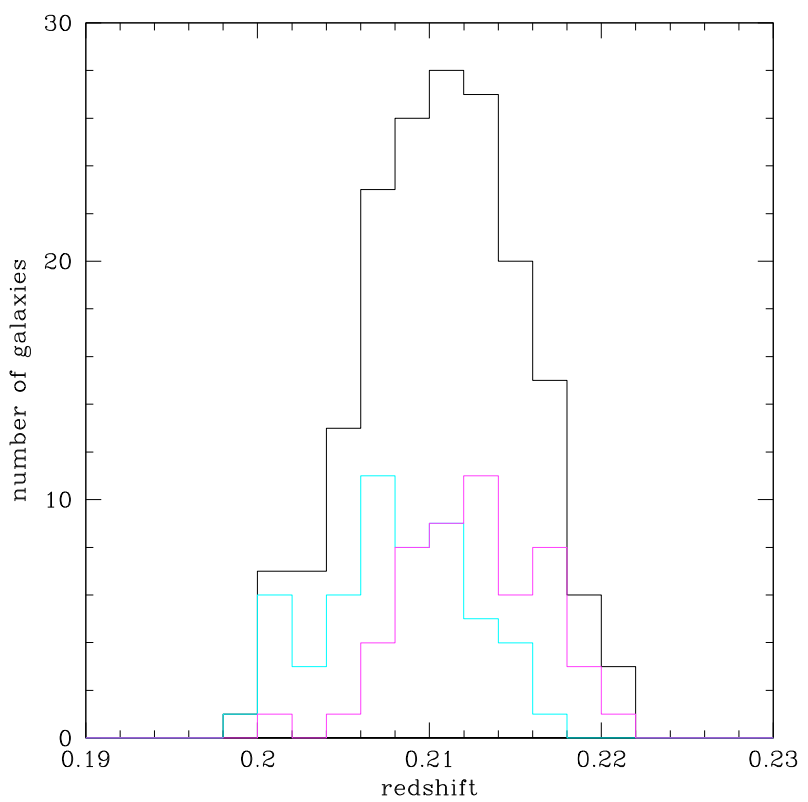

Fig. 12. Redshift histograms for all the galaxies with measured redshifts in the regions covered by Abell 222 and Abell 223 (in black), and for the galaxies in the gravitationally bound structures of Abell 222 and Abell 223, in magenta and cyan respectively (the positions of these galaxies are shown in Fig. 11) with the same colour coding.

corresponding velocity dispersions are 1014 and $1170 \mathrm{~km} \mathrm{~s}^{-1}$ respectively. The virial masses derived for these two substructures (assuming $M / L=400$ solar units) are $1.9 \times 10^{11}$ and $4.2 \times 10^{13} M_{\odot}$.

For Abell 222, and probably also for Abell 223, these masses are obviously underestimated, particularly for Abell 222, since such a value does not even reach that of a $\mathrm{cD}$ galaxy. We have tried to analyze the two clusters separately, but this modifies the resulting masses only slightly. The fact that we find too small cluster masses can be due to several reasons. First, the redshift sample we are using is taken from the NED data base, and is obviously not complete, even at bright magnitudes. We have estimated the completeness of the redshift catalogue by comparing the numbers of galaxies with redshifts in the $[0.18,0.24]$ redshift interval to the total number of galaxies, in magnitude bins of 0.5 in the $r^{\prime}$ band. We find that for $17<r^{\prime}<19.5$ the completeness is around $50 \%$, then rapidly decreases for $r^{\prime}>19.5$, confirming that the cluster masses based on such a redshift catalogue are probably unreliable. Second, as pointed out by the referee, the SG method relies on the unlikely assumption that galaxies in clusters are the mass carriers, and additionally requires an assumption about the $M / L$ ratio, here taken to be $M / L=400$; this value may be reasonable for a cluster taken as a whole, but is too large for individual galaxies (the dark matter halos of individual galaxies would be overlapping in the dense central regions of clusters, and strong mass segregation would result by dynamical friction, which is not observed).

Better estimates of the cluster masses can be obtained by applying other methods. For example, the relation between mass and velocity dispersion computed by Biviano et al. (2006) leads to masses of $1.2 \times 10^{15}$ and $1.4 \times 10^{15} M_{\odot}$ (with $H_{0}=70 \mathrm{~km} \mathrm{~s}^{-1} \mathrm{Mpc}^{-1}$ ) for Abell 222 and Abell 223 respectively, based on respective velocity dispersions of 1014 and $1070 \mathrm{~km} \mathrm{~s}^{-1}$. Note however that since the clusters are not virialized these values are probably overestimates. For example, Takizawa et al. (2010) have recently shown from numerical

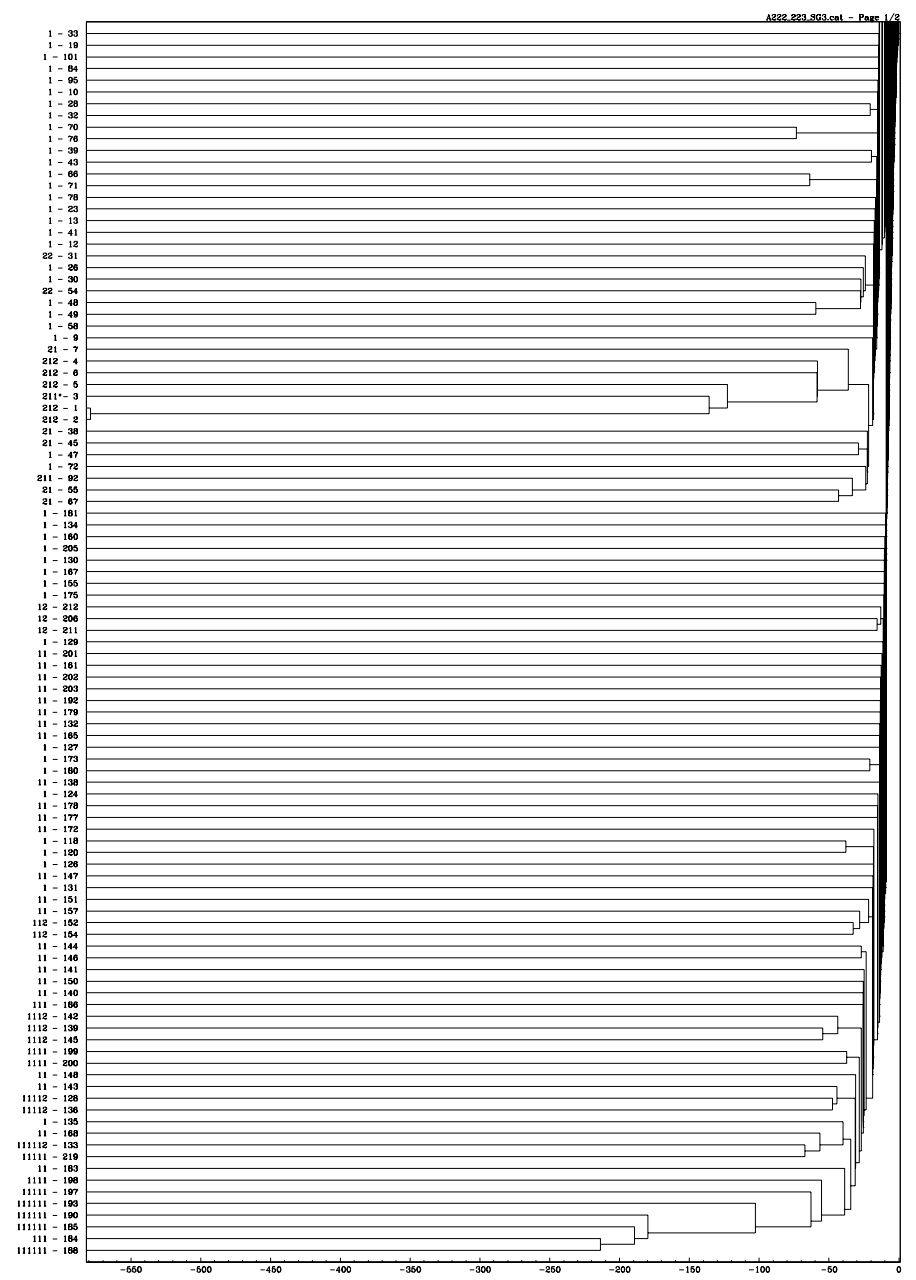

Fig. 13. Dendogram showing how Abell 222 and Abell 223 can be dynamically separated when applying the Serna \& Gerbal algorithm. The abscissa is the negative binding energy while the catalogue numbers of the various galaxies are shown along the ordinate (the top of the dendogram is truncated, but would only show a constant gravitational binding energy).

simulations that for merging systems where the subcluster has a mass larger than one fourth that of the large cluster, the virial mass can be larger than twice the real mass. This most probably applies at least to the Abell 223 cluster.

We can also apply the mass-temperature relation estimated from X-ray data by Arnaud et al. (2005), with the overall temperatures given in Table 5 ; this relation gives masses $M_{200}$ of $3.8 \times 10^{14}$ and $4.7 \times 10^{14} M_{\odot}$ for Abell 222 and Abell 223 respectively (also with $H_{0}=70 \mathrm{~km} \mathrm{~s}^{-1} \mathrm{Mpc}^{-1}$ ). These values may be closer to the real values than those estimated from the galaxy velocity dispersions, since e.g. Takizawa et al. (2010) found that for merging clusters X-ray derived masses were usually more reliable than virial mass estimations. However, as explained for example by Nagai et al. (2007), although the total ICM mass can be measured quite accurately (to better than $\sim 6 \%$ ) in clusters, the hydrostatic estimate of the gravitationally bound mass is biased low by about 5-20\% throughout the virial region, primarily due to additional pressure support provided by subsonic bulk motions in the ICM, ubiquitous in our simulations even in relaxed systems. Another source of error is the fact that the cluster temperatures, particularly that of Abell 223 (see Fig. 18) are not homogeneous. For example, Rasia et al. (2004) have shown that an incomplete thermalisation of the gas due to an incomplete 


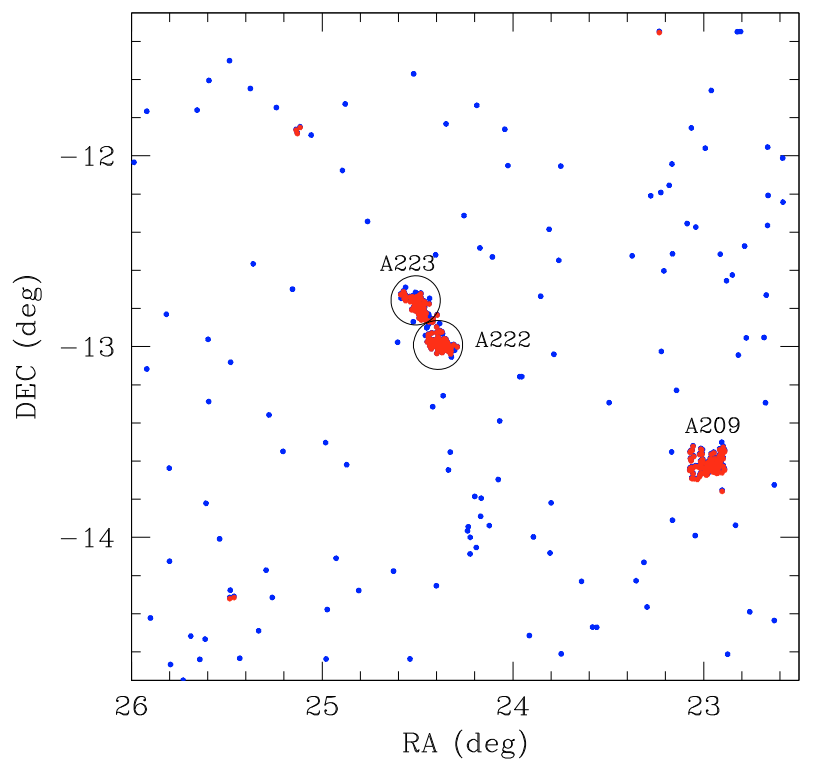

Fig. 14. Distribution of the galaxies with available redshifts in a large region surrounding the clusters. Blue points: all galaxies with measured redshifts, red points: galaxies with redshifts in the $[0.18,0.24]$ interval. The two clusters under study are circled. A third cluster appears to the southwest: Abell 209. The physical size of this map is $44.7 \times 44.7 \mathrm{Mpc}^{2}$.

virialisation of the cluster could lead to an underestimate of the mass. On the other hand, if the X-ray gas is heated by a collision, the mass could be overestimated.

We can also note that Mamon (1993) has calculated the bias on the $M / L$ ratio when the virial theorem is wrongly applied to a group which is still evolving dynamically (see his Fig. 11). He found that in some cases the mass could be underestimated by a factor reaching 100. Therefore the masses estimated for our clusters assuming that they are virialized are clearly not valid.

Unfortunately, the SG method does not allow to separate the two substructures in Abell 223 discovered by Dietrich et al. (2002) based on galaxy density contours (see their Fig. 6).

Note that Abell 223 is probably close to the intersection of two or more filaments, since it is massive and substructured, and its GLF is comparable to that e.g. of Coma (see Sect. 3.3). On the other hand, Abell 222 is probably only following its path along a filament.

\subsection{Large-scale structure}

Out of the 980 galaxies found in NED in a 3 deg radius region, 310 have redshifts in the $[0.18,0.24]$ interval (i.e. about $\pm 9000 \mathrm{~km} \mathrm{~s}^{-1}$ around the mean cluster velocities). Their spatial distribution is shown in Fig. 14. A cluster is visible towards the southwest: Abell 209. It has a redshift similar to that of Abell 222 and Abell 223 and is located about 19.2 Mpc away from Abell 222 in projection on the sky. No significant filamentary structure is detected towards the Abell 222/223 clusters.

\section{X-ray analysis}

\subsection{Data reduction}

The pair of clusters Abell 222/223 was observed with XMM-Newton in two different pointings (revolutions 1378 and 1380) with a total exposure time of $144 \mathrm{ks}$. We used data from all EPIC cameras (MOS1, MOS2 and pn). The data were reduced with the XMM-Newton Science Analysis System (SAS)
Table 4. Information on X-ray data.

\begin{tabular}{llllll}
\hline \hline Name & Filter & \multicolumn{2}{c}{$\begin{array}{c}t_{\text {exp }}(\mathrm{ks}) \\
\text { MOS2 }\end{array}$} & pn & $n_{\mathrm{H}}\left(10^{20} \mathrm{~cm}^{-2}\right)$ \\
\hline Abell 222 & Thin1 & 11.814 & 18.005 & 10.226 & 2.26 \\
Abell 223 & Thin1 & 23.479 & 26.069 & 10.612 & 2.26 \\
\hline & & & & \\
\hline
\end{tabular}

Fig. 15. Annulus where the background was extracted for subtraction.

Table 5. Overall X-ray temperatures and metallicities.

\begin{tabular}{cccc}
\hline \hline Cluster & $\begin{array}{c}r_{200} \\
(\mathrm{Mpc})\end{array}$ & $\begin{array}{c}k T \\
(\mathrm{keV})\end{array}$ & $\begin{array}{c}Z \\
(\text { solar })\end{array}$ \\
\hline Abell 222 & $1.28 \pm 0.11$ & $3.77 \pm 0.15$ & $0.23 \pm 0.06$ \\
Abell 223 & $1.55 \pm 0.15$ & $4.38 \pm 0.16$ & $0.23 \pm 0.08$ \\
\hline
\end{tabular}

v8.0 and calibration database with all updates available prior to November 2009. The initial data screening was applied using recommended sets of event patterns, 0-12 and 0-4 for the MOS and PN cameras, respectively.

The light curves are not constant and large variations in intensity are visible (flares). To improve the signal-to-noise ratio we discarded periods of flares and the cleaned light curves in the energy range of [1-10] keV exhibited stable mean count rates; exposure times are given in Table 4 . We considered events inside the field of view (FOV) and excluded all bad pixels.

The background was taken into account by extracting MOS1, MOS2 and pn spectra from the publicly available EPIC blank sky templates of Andy Read (Read \& Ponman 2003). The background was normalized using a spectrum obtained in an annulus (between 12.5-14 arcmin) where the cluster emission is no longer detected. In one of the pointings, the A223 cluster falls exactly inside the annulus where we determine the background contribution. We thus masked this region, avoiding any cluster contamination. In Fig. 16 we show a comparison between the observed and Read backgrounds for the three detectors, as well as the residuals between both determinations.

In order to determine the global properties of these clusters, we adopted the virial radii $r_{200}$ determined by the weak lensing analysis (Dietrich et al. 2005). These values, together with the mean temperatures and metallicities are given in Table 5. They were calculated fixing the hydrogen column density to its galactic value of $n_{\mathrm{H}}=1.56 \times 10^{20} \mathrm{~cm}^{-2}$. 

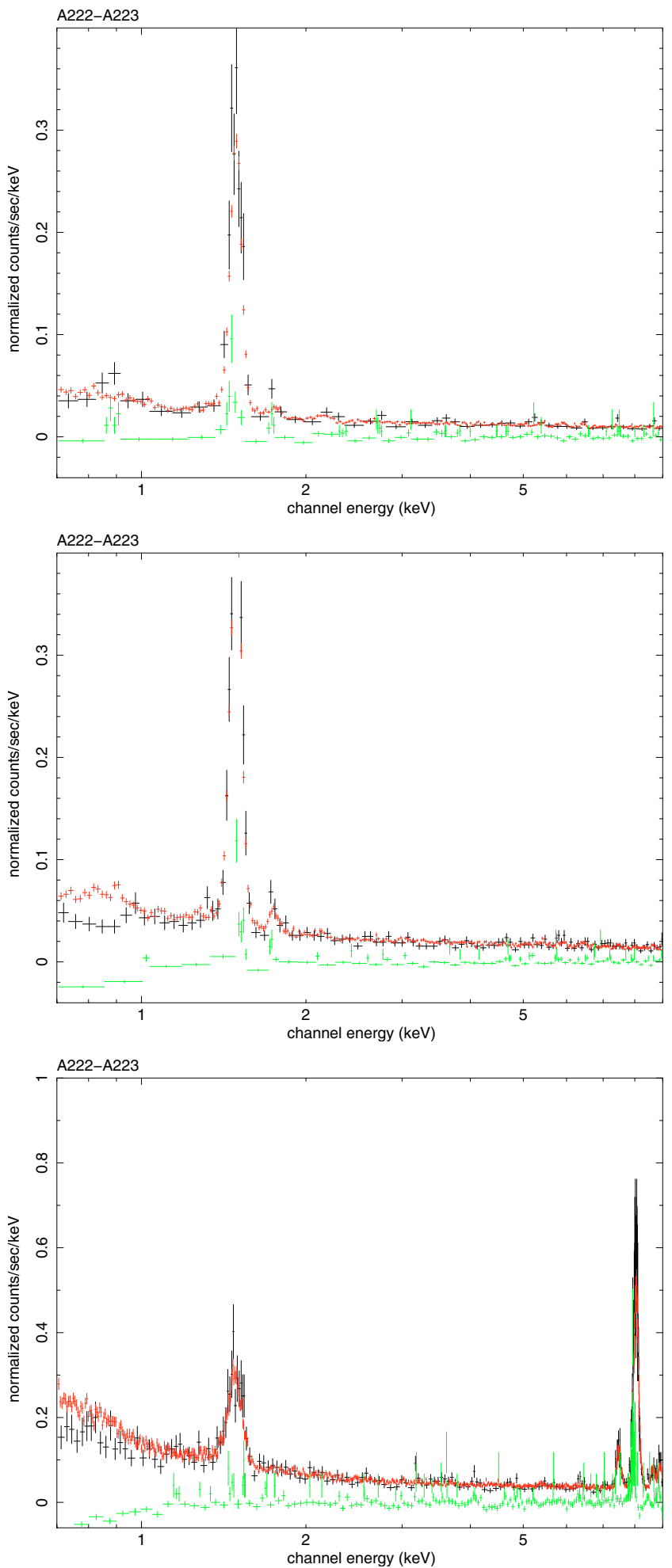

Fig. 16. Comparison of the observed and Read backgrounds for MOS1 (top panel), MOS2 (middle panel) and pn (low panel). The black points correspond to the observed data, the red points to Read's scaled background and the green points to the residuals.

\subsection{Spectrally measured $2 D X$-ray maps}

We performed quantitative studies using X-ray spectrally measured 2D maps to derive global properties of these clusters. These maps were made in a grid; for each spatial bin we set a minimum count number of 900 (after background subtraction).
For the spectral fits, we used XSPEC version 11.0.1 (Arnaud 1996) and modeled the obtained spectra with a MEKAL single temperature plasma emission model (bremsstrahlung + line emission, Kaastra \& Mewe 1993; Liedahl et al. 1995). The free parameters are the X-ray temperature $(\mathrm{kT})$ and the metal abundance (metallicity). Spectral fits were made in the energy interval of [0.7-8.0] keV with the hydrogen column density fixed at the galactic value (see Table 4), estimated with the $\mathrm{nH}$ task of FTOOLS (based on Dickey \& Lockman 1990).

We compute the effective area files (ARFs) and the response matrices (RMFs) for each region in the grid. This procedure (already presented in Durret et al. 2005; and Laganá et al. 2008) allows us to perform a reliable spectral analysis in each spatial bin, in order to derive high precision metallicity and temperature maps (see Fig. 17), since we simultaneously fit all three instruments for both pointings (that is, we coadd 6 spectra: 2 MOS1, 2 MOS2 and 2 PN). The best fit value is then attributed to the central pixel.

The resulting X-ray temperature and metallicity maps are displayed in Figs. 18 and 19. Maps of the errors on these two maps are given in the Appendix (Figs. B.1 and B.2).

The study of the thermal structure of the intra-cluster medium (ICM) provides a very interesting record of the dynamical processes that clusters of galaxies have experienced during their formation and evolution. The temperature (and pressure) distribution of the ICM gives us important insight into the process of galaxy cluster merging and on the dissipation of the merger energy in form of turbulent motion. Metallicity maps can be regarded as a record of the integral yield of all the different stars that have released their metals through supernova explosions or winds during cluster evolution.

From the temperature maps we can see that neither cluster presents a defined cool-core as a sign of relaxation. However, Abell 222 is fairly isothermal when compared to Abell 223. The overall temperature structure in Abell 223 shows the clear imprint of the recent interaction(s) this cluster has been through. The cluster exhibits a remarkable blob of hotter gas at its southwest extremity, a colder region to the southeast, and a hotter zone crossing the cluster along a roughly east-west direction. The metallicity map of Abell 222 is quite homogeneous, while that of Abell 223 is very inhomogeneous, with a metallicity enhancement in a zone which does not completely coincide with the colder southeast region and a low metallicity region extending along a zone curving from the east to the west-northwest.

\subsection{Interpretation}

As has been shown by Kapferer et al. (2006, see his Fig. 10), the enhancement of the metallicity from $Z=0.2 Z_{\odot}$ (in the region of the shock) to $Z=0.4 Z_{\odot}$ (in the surrounding region) takes 3 Gyr (assuming their model B). This means that the gas has not yet had time to mix and distribute the metals in the region where the shock is still propagating. As can be seen in the larger variations in metallicity that Abell 223 presents when compared to Abell 222, the metallicity of merging systems is not smoothly distributed. It is likely that Abell 223 has recently been crossed by a smaller cluster, which now appears at the north east tip of the maps.

From the metallicity map we also observe that the central region of Abell 223 presents a metallicity enhancement when compared to the surrounding medium, though not highly centrally peaked. At this point, it is important to highlight that Abell 223 has more very bright galaxies than Abell 222. The results of a former work (Laganá et al. 2009) suggested that BCGs alone 


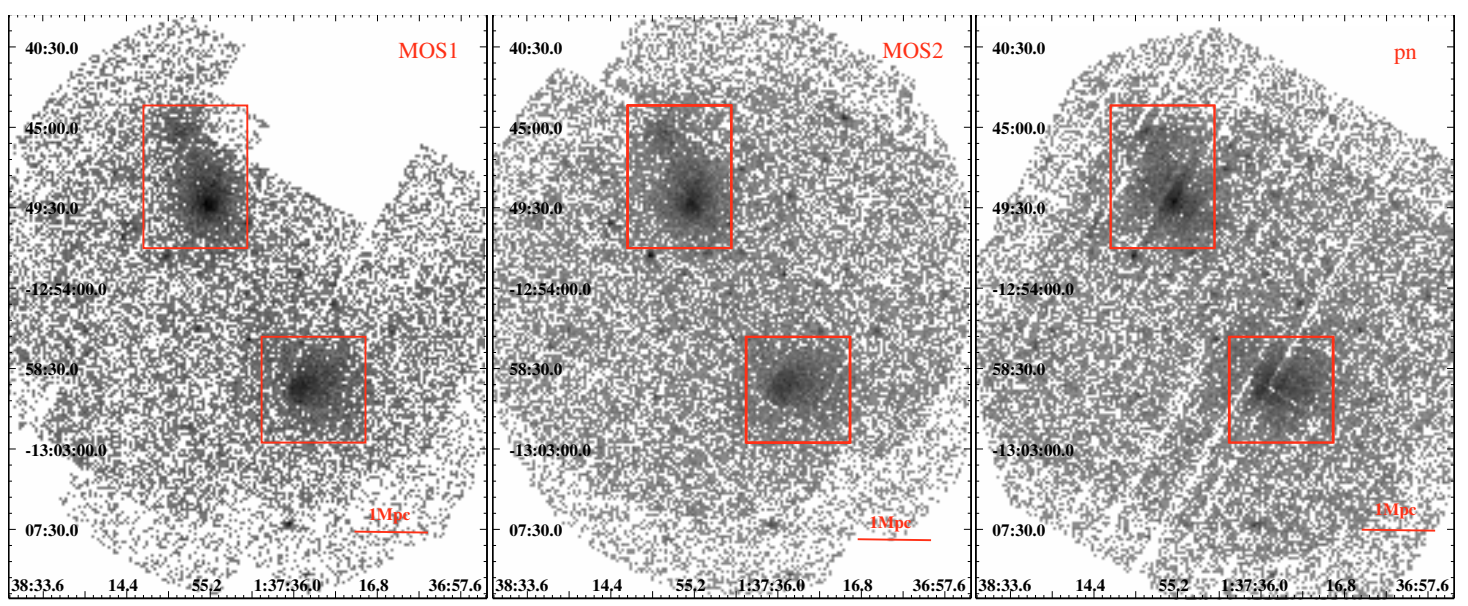

Fig. 17. Merged event maps from MOS1 (left panel), MOS2 (central panel) and pn (right panel) showing the regions where the 2D maps were obtained.

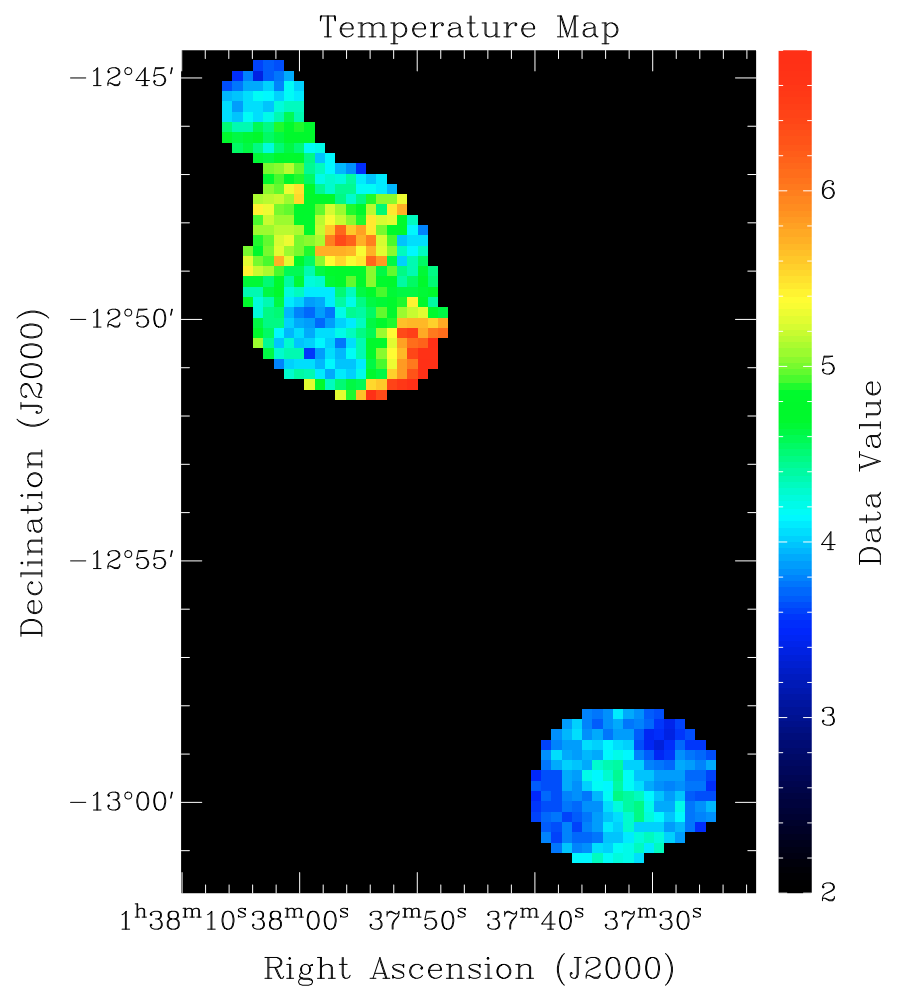

Fig. 18. X-ray temperature map for Abell 222 (bottom) and Abell 223 (top). The color-bar indicates the temperature in $\mathrm{keV}$. The corresponding error map is shown in Fig. B.1.

cannot produce the observed metallicity excess in the central region (De Grandi \& Molendi 2001; De Grandi et al. 2004). Thus, it is likely that the cool-core has been disrupted by the sub-cluster collision but the metals injected into the ICM by very bright galaxies of Abell 223 have not moved that much away from the central region yet. This can occur if one assumes ram-pressure stripping with tidal disruption of these very bright galaxies near the center as a mechanism for metal injection. In this case, we would expect Abell 223 to have a central metallicity higher than Abell 222, and this is indeed the case.

It is interesting to note that Abell 223 has in fact two BCGs. The galaxy that we consider as being in the cluster center looks like a $\mathrm{cD}$, with several smaller satellite galaxies. The surface brightness profiles of the brightest galaxies of Abell 223 are

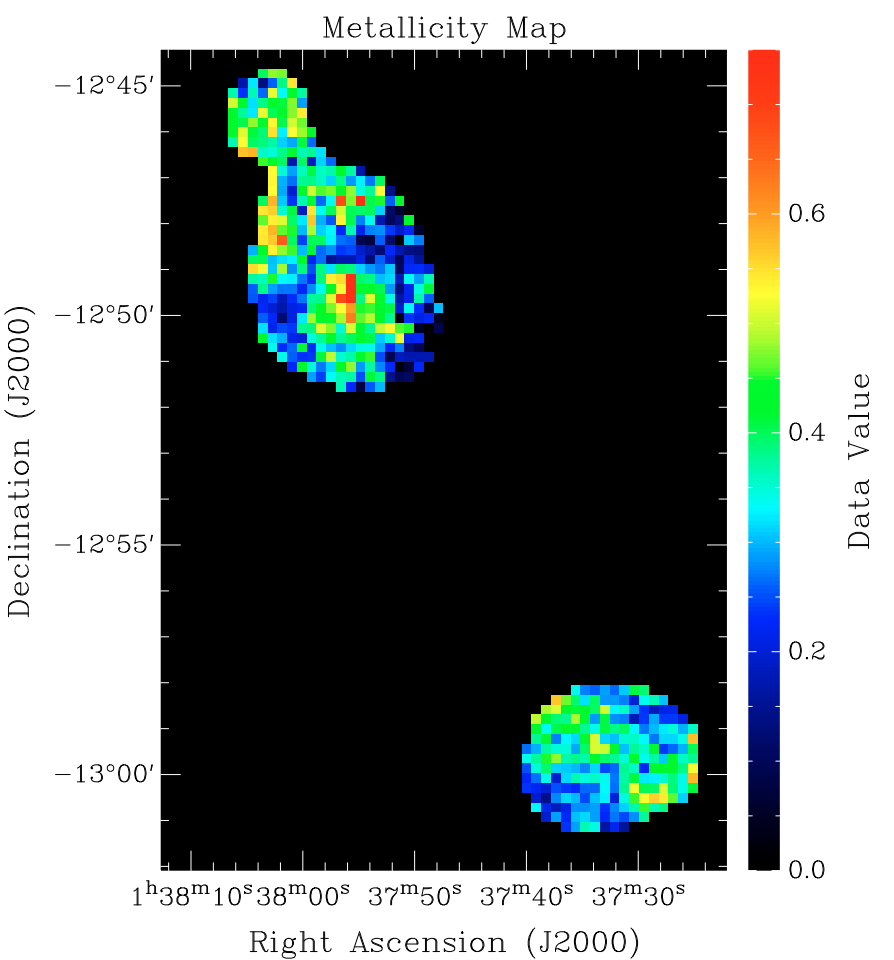

Fig. 19. X-ray metallicity map for Abell 222 (bottom) and Abell 223 (top). The color-bar indicates the metallicity in solar units. The corresponding error map is shown in Fig. B.2.

displayed in Fig. 20. We can see that the surface brightness profile of the "cD" decreases slowlier than those of the other bright galaxies. It can therefore be considered as a dominant galaxy, since it also shows clear signs of an associated population of fainter galaxies in the likely process of being accreted. A second nearby elliptical galaxy which is even brighter could be the central galaxy of an accreted group. This is reminiscent of the Coma cluster where the "cD" (NGC 4874) is not quite as bright as the brightest galaxy (NGC 4889), which is believed to have been accreted after the cluster was formed.

In the case of Abell 222, the situation is even more complicated, since there are three brightest cluster galaxies, aligned along the direction seen in Fig. 11 and having exactly the same magnitude, the middle one being surrounded by several small satellite galaxies that give it the appearance of a $\mathrm{cD}$. This is 


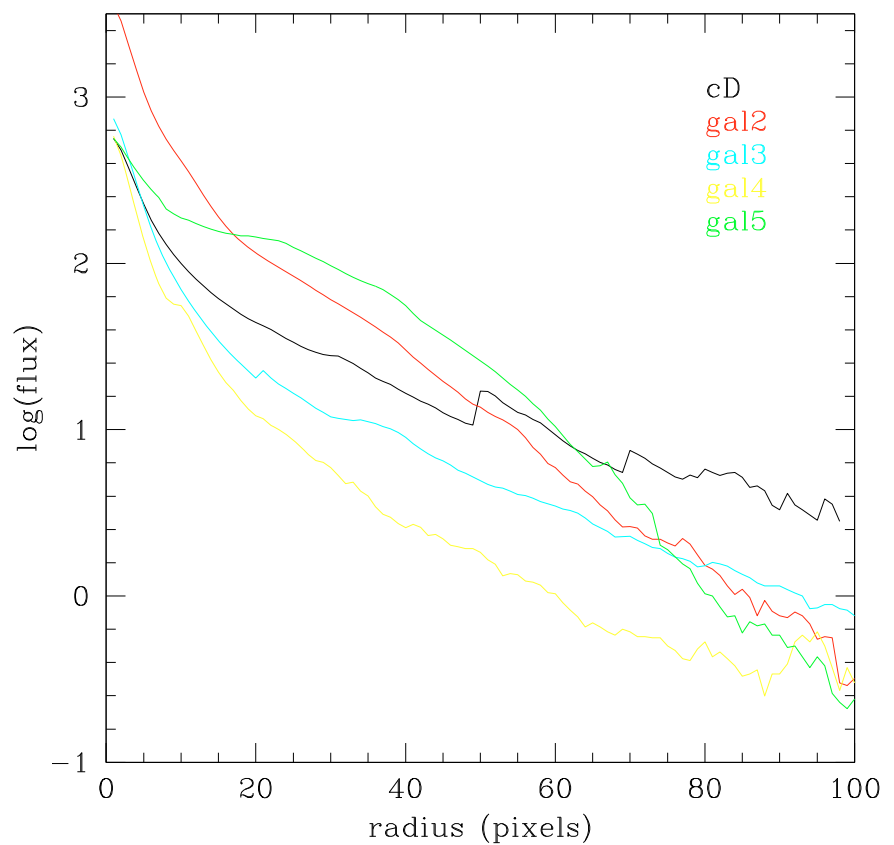

Fig. 20. Surface brightness profiles of the five brightest galaxies in Abell 223. The "cD" is in black, the very bright galaxy near the $\mathrm{cD}$ is in red, and the three other galaxies are somewhat more distant from the cluster center. The $\mathrm{cD}$ profile decreases notably slower with radius that the profiles of the other galaxies.

another argument in favour of the idea that Abell 222 is not fully relaxed either.

\section{Discussion and conclusions}

Understanding the interplay between galaxies and ICM is fundamental for further investigations of galaxy clusters. In this way, an important step is made with multiwavelength studies of these structures.

In order to understand better the connection between ICM and galaxies in merging systems we combined both X-ray and optical data to examine in detail the pair of clusters Abell 222/Abell 223. To do so, we combined spectroscopically computed 2D-maps with optical data to gain insight on the cluster history. We verified that besides their proximity, these clusters show very different evolutionary stages that can be detected in optical and X-ray analyses. The Abell 223 data indicate that this cluster is dynamically perturbed when compared to its companion. Signs of recent merger(s) are detected at both wavelengths, meaning that the gas and the galaxies are still out of equilibrium. It is more likely that the signs of merger detected in Abell 223 are due to the interaction with the subcluster. On the other hand, Abell 222 is a smaller cluster closer to a stable dynamical state than Abell 223.

As mentioned in Sect. 5.2, Abell 223 exhibits a remarkable blob of hotter gas at its southwest extremity. The gas of this region may have been compressed and heated by a recent shock that this cluster has been through. Another zone of higher temperature is elongated towards the north east-south west direction. This is close to the direction that connects the two clusters and where a filament of low temperature was already detected between these clusters (Werner et al. 2008). This region does not appear in our 2D maps because there are not enough X-ray counts. However, the X-ray contours overlaid in the 2D maps show that the emission goes beyond the limits of the maps and towards the region where the filament is located.

The presence of only a small number of hot blobs and the low X-ray temperature of the filament tend to indicate that this system is in the first stages of a merger. This hints at a scenario in which the two clusters are at the first stages of a collision. These clusters are beginning to collide and from the optical counterpart analysis, the central region of Abell 223 already exhibits signs of this interaction presenting a higher number of star forming galaxies. So it seems probable that a small cluster has just crossed Abell 223 and is heading towards the north east. On the other hand, Abell 222 does not present strong evidence for interaction other than the absence of a cool-core.

We thus summarize our main results below:

- From the optical analysis we conclude that Abell 222 is smaller and less massive than Abell 223. For Abell 222, the GLF is quite well fit by a Schechter function in both the $r^{\prime}$ and $g^{\prime}$ bands. On the other hand, since Abell 223 is a double cluster, it presents a "perturbed" GLF. Abell 223 has more bright galaxies than Abell 222 and as a consequence a second component is required to fit the GLF at bright magnitudes. The GLFs of both clusters are comparable in the $r^{\prime}$ band but in the $g^{\prime}$ band the GLF is notably steeper in Abell 223 than in Abell 222. This is explained by a higher number of faint star forming galaxies present in Abell 223. Star formation is triggered by recent merger events. Such mergers disturbed the gas and their imprints can be observed in 2D X-ray maps.

- Both clusters appear to be elongated when traced by their cluster galaxy distributions. This is another evidence that these clusters are not in equilibrium. However, the X-ray filament joining the two clusters does not seem to play a very important role in the evolution of cluster galaxies. This is probably explained by the low temperature of the gas, since ram-pressure is not very efficient below $2 \mathrm{keV}$.

- From the spectrally measured 2D X-ray maps we conclude that Abell 222 is fairly isothermal when compared to Abell 223. However, neither of these clusters presents a coolcore as a sign of relaxation. The overall temperature map of Abell 223 is an imprint of the recent dynamical interactions this cluster has experienced. The broad variation in temperature puts in evidence a large number of sub-structures. From the metallicity map, we also observe an enhancement in the central region of Abell 223. This can be explained by the fact that this cluster has more very bright galaxies than Abell 222. Assuming ram-pressure stripping with tidal disruption of the bright galaxies near the cluster center as a mechanism for metal injection, one would expect this cluster to have a higher central metallicity than Abell 222. The combined analysis of X-ray and optical data argues for a scenario in which the two clusters are beginning to collide. The former cluster presents signs of interaction in both wavelength ranges, and has probably been crossed by a small cluster heading north east, while its companion does not show strong evidence for interaction, except for the absence of a cool-core.

Acknowledgements. We are grateful to G. B. Lima Neto for his help in obtaining the X-ray maps. We warmly thank Andrea Biviano for giving us his Schechter function fitting programme free of charge and helping us with the corresponding plots. We also wish to thank Delphine Russeil for pointing the fact that the clusters under study are in the direction of the Sagittarius stream, and Stephen Gwyn for email exchanges concerning the Megapipe archive. Last but not least, we warmly thank the referee, Andrea Biviano, for his relevant questions and constructive comments. This work was supported by FAPESP (grants: 2006/562139, 2008/04318-7). 


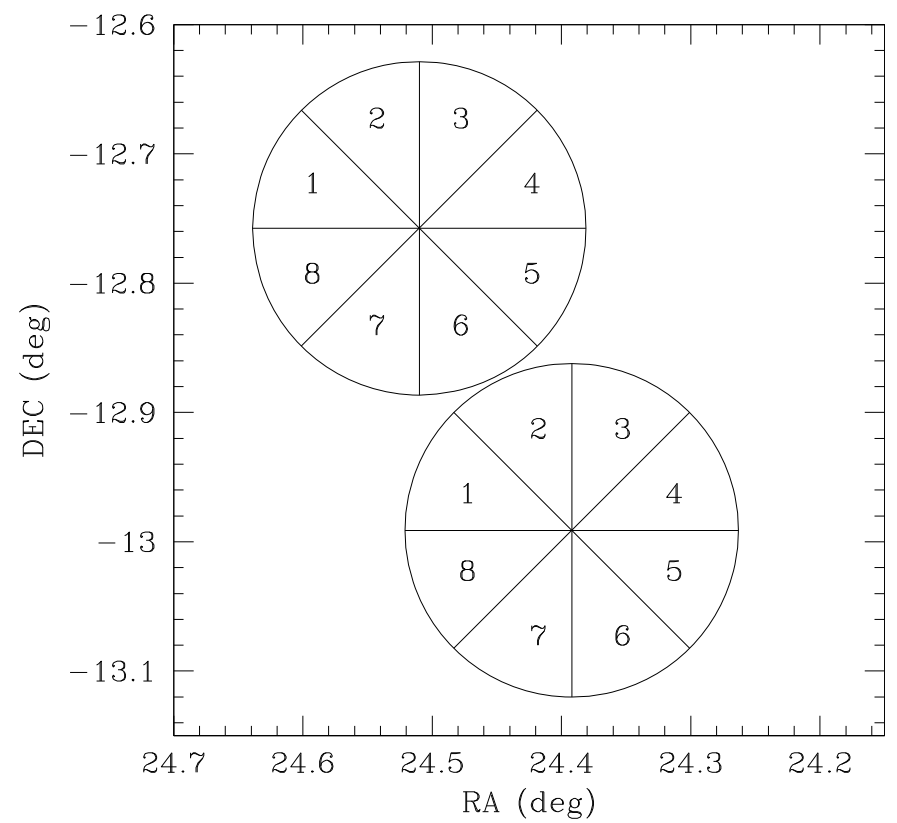

Fig. A.1. Positions of the sectors used to draw Fig. 9.

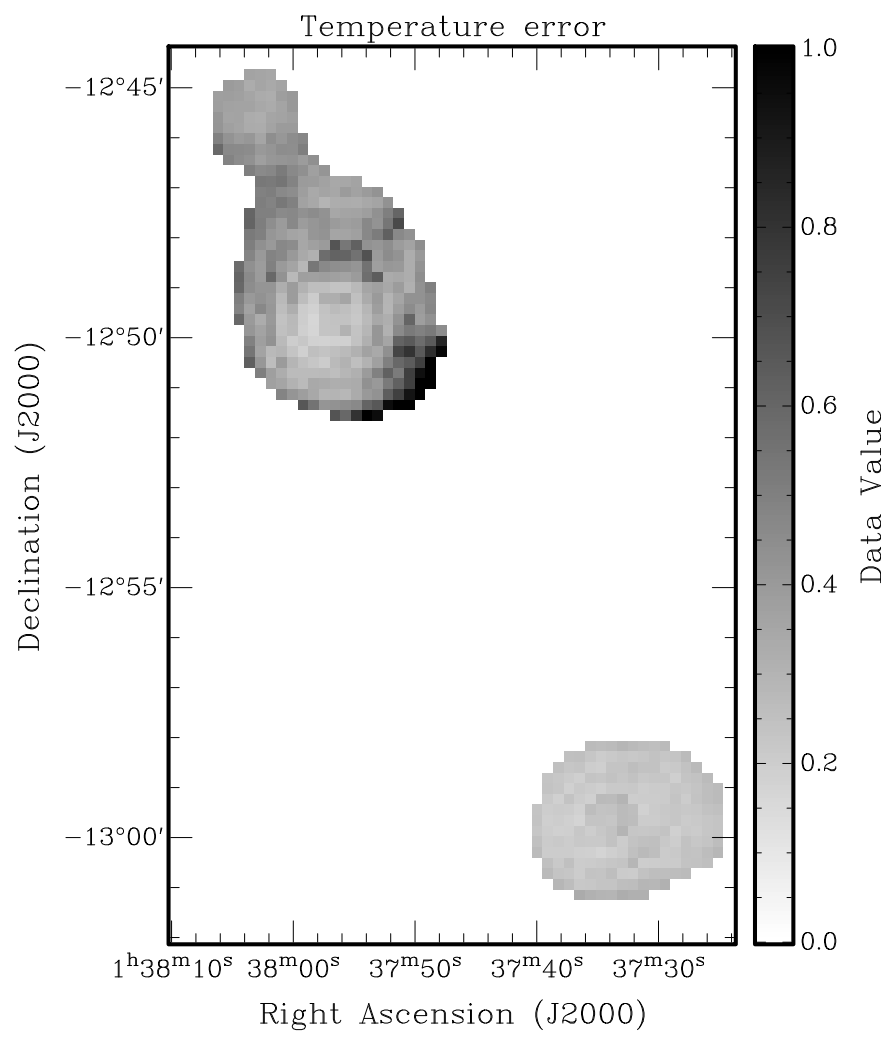

Fig. B.1. Error map on the X-ray temperature.

\section{Appendix A: Sectors for Fig. 9}

The positions of the sectors used to draw Fig. 9 are shown in Fig. A.1.

\section{Appendix B: Error maps}

The maps of the errors on the X-ray temperature and metallicity maps are displayed in Figs. B.1 and B.2. The errors on these

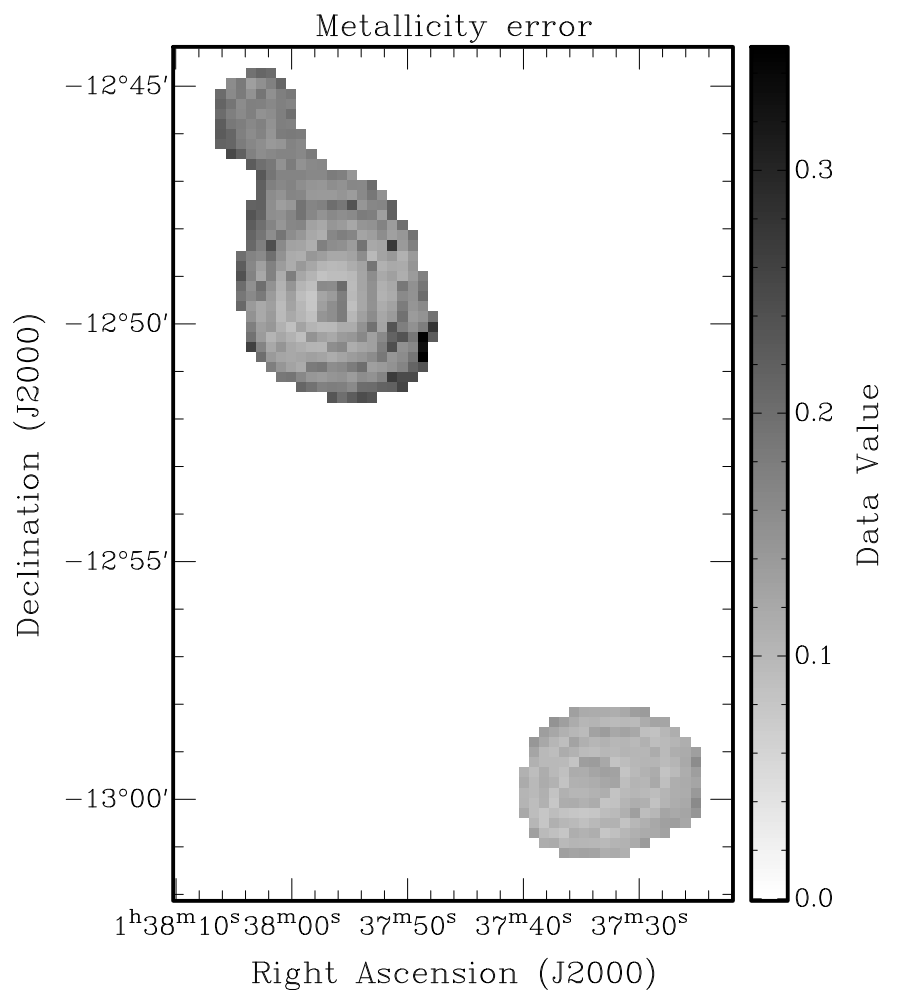

Fig. B.2. Error map on the X-ray metallicity.

parameters were directly obtained from the spectral fits and they give the deviations in the maps presented in Figs. 18 and 19.

\section{References}

Adami, C., Biviano, A., \& Mazure, A. 1998, A\&A, 331, 439 Adami, C., Picat, J.-P., Savine, C., et al. 2006, A\&A, 451, 1159 Andreon, S., Punzi, G., \& Grado, A. 2005, MNRAS, 360, 727

Andreon, S., Puddu, E., de Propris, R., \& Cuillandre, J.-C. 2008, MNRAS, 385, 979

Arnaud, K. A. 1996, ASPC, 101, 17

Arnaud, M., Pointecouteau, E., \& Pratt, G. W. 2005, A\&A, 411, 893

Bertin, E., \& Arnouts, S. 1996, A\&AS, 117, 393

Biviano, A., Durret, F., Gerbal, D., et al. 1995, A\&A, 297, 610

Biviano, A., Katgert, P., Mazure, A., et al. 1997, A\&A, 321, 84

Biviano, A., Murante, G., Borgani, S., et al. 2006, A\&A, 456, 23

Boschin, W., Girardi, M., Barrena, R., et al. 2004, A\&A, 416, 839

Boué, G., Adami, C., Durret, F., Mamon, G., \& Cayatte, V. 2008, A\&A, 479, 335

De Grandi, S., \& Molendi, S. 2001, ApJ, 551, 153

De Grandi, S., Ettori, S., Longhetti, M., \& Molendi, S. 2004, A\&A, 419, 7

Dickey, J. M., \& Lockman, F. J. 1990, ARA\&A, 28, 215

Dietrich, J. P., Clowe, D. I., \& Soucail, G. 2002, A\&A, 394, 395

Dietrich, J. P., Schneider, P., Clowe, D., Romano-Díaz, E., \& Kerp, J. 2005, A\&A, 440, 453

Dressler, A., \& Shectman, S. A. 1988, AJ, 95, 985

Durret, F., Lima Neto, G. B., \& Forman, W. 2005, A\&A, 432, 809

Fadda, D., Biviano, A., Marleau, F. R., Storrie-Lombardi, L. J., \& Durret, F. 2008, ApJ, 672, L9

Girardi, M., \& Mezzetti, M. 2001, ApJ, 548, 79

Girardi, M., Boschin, W., \& Barrena, R. 2006, A\&A, 455, 45

Gwyn, S. D. J. 2009, ADASS XVIII, ASP Conf. Ser., 411, 123

Hoyle, F., \& Vogeley, M. S. 2004, ApJ, 607, 751

Ibata, R., Irwin, M., Lewis, G. F., \& Stolte, A. 2001, ApJ, 547, L133

Ilbert, O., Tresse, L., Zucca, E., et al. 2005, A\&A, 439, 863

Kaastra, J. S., \& Mewe, R. 1993, A\&AS, 97, 443

Kapferer, W., Ferrari, C., Domainko, W., et al. 2006, A\&A, 447, 827

Laganá, T. F., Lima Neto, G. B., Andrade-Santos, F., \& Cypriano, E. S. 2008, A\&A, 485, 633

Laganá, T. F., Dupke, R. A, Sodré, Jr., L., et al. 2009, MNRAS, 394, 357 
Liedahl, D. A., Osterheld, A. L., \& Goldstein, W. H. 1995, ApJ, 438, L115 Mamon, G. 1993, in Proc. Aussois meeting, March 21-25, ed. F. Combes, \& E. Athanassoula (Observatoire de Paris-Meudon), 188

[arXiv: astro-ph/9308032]

Murante, G., Giovalli, M., Gerhard, O., et al. 2007, MNRAS, 377, 2

Nagai, D., Vikhlinin, A., \& Kravtsov, A. V. 2007, ApJ, 655, 98

Peng, C. Y., Ho, L. C., Impey, C. D., \& Rix, H. W. 2002, AJ, 124, 266

Poggianti, B. M., Bridges, T. J., Komiyama, Y., et al. 2004, ApJ, 601, 197

Proust, D., Cuevas, H., Capelato, H. V., et al. 2000, A\&A, 355, 443

Rasia, E., Tormen, G., \& Moscardini, L. 2004, MNRAS, 351, 237

Read, A. M., \& Ponman, T. J. 2003, A\&A, 409, 395
Richards, G. T., Strauss, M. A., Fan, X., et al. 2006, AJ, 131, 2766

Robin, A. C., Reylé, C., Derrière, S., \& Picaud, S. 2003, A\&A, 409, 523 Roettiger, K., Burns, J. O., \& Loken, C. 1996, ApJ, 473, 651

Schlegel, D. J., Finkbeiner, D. P., \& Davis, M. 1998, ApJ, 500, 525 Serna, A., \& Gerbal, D. 1996, A\&A, 309, 65

Takizawa, M., Nagino, R., \& Matsushita, K. 2010, PASJ, accepted [arXiv: 1004.3322]

Werner, N., Finoguenov, A., Kaastra, J. S., et al. 2008, A\&A, 482, L29 Wright, E. L. 2006, PASP, 118, 1711

Yanny, B., Newberg, H. J., Johnson, J. A., et al. 2009, ApJ, 700, 1282

Zabludoff, A. I., \& Zaritsky, D. 1995, ApJ, 447, 21 